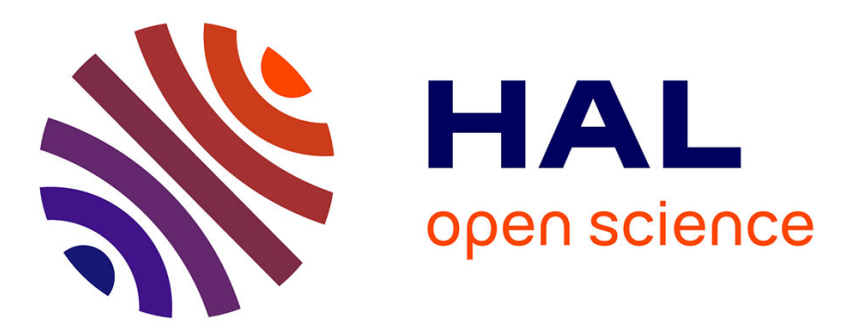

\title{
A simple pseudospectral method for the computation of the time-dependent Dirac equation with Perfectly Matched Layers
}

\author{
Xavier Antoine, Emmanuel Lorin
}

\section{- To cite this version:}

Xavier Antoine, Emmanuel Lorin. A simple pseudospectral method for the computation of the timedependent Dirac equation with Perfectly Matched Layers. Journal of Computational Physics, 2019, 395, pp.583-601. 10.1016/j.jcp.2019.06.020 . hal-02340843

\section{HAL Id: hal-02340843 \\ https://hal.science/hal-02340843}

Submitted on 31 Oct 2019

HAL is a multi-disciplinary open access archive for the deposit and dissemination of scientific research documents, whether they are published or not. The documents may come from teaching and research institutions in France or abroad, or from public or private research centers.
L'archive ouverte pluridisciplinaire HAL, est destinée au dépôt et à la diffusion de documents scientifiques de niveau recherche, publiés ou non, émanant des établissements d'enseignement et de recherche français ou étrangers, des laboratoires publics ou privés. 


\title{
A simple pseudospectral method for the computation of the time-dependent Dirac equation with Perfectly Matched Layers
}

\author{
Xavier ANTOINE ${ }^{\mathrm{a}}$, Emmanuel LORIN ${ }^{\mathrm{b}, \mathrm{c}}$ \\ ${ }^{a}$ Institut Elie Cartan de Lorraine, Université de Lorraine, UMR 7502, Inria Nancy-Grand Est, F-54506 \\ Vandoeuvre-lès-Nancy Cedex, France \\ ${ }^{b}$ School of Mathematics and Statistics, Carleton University, Ottawa, Canada, K1S 5B6 \\ ${ }^{c}$ Centre de Recherches Mathématiques, Université de Montréal, Montréal, Canada, H3T 1J4
}

\begin{abstract}
A simple time-splitting pseudospectral method for the computation of the Dirac equation with Perfectly Matched Layers is proposed. Within this approach, basic and widely used FFT-based solvers can be adapted without much effort to compute Initial Boundary Value Problems for the time-dependent Dirac equation with absorbing boundary layers. Some numerical examples from laser-physics are proposed to illustrate the method.
\end{abstract}

Keywords: Dirac equation; time-splitting; pseudospectral approximation; Perfectly Matched Layers; high-order accuracy

\section{Introduction}

This paper is concerned with the numerical computation of the time-dependent Dirac equation for physical problems involving delocalized wavefunctions. More specifically, we are interested in the numerical solution to the Dirac equation on a truncated domain with absorbing boundary layers. To this end, we propose a simple combination of a pseudospectral method with Perfectly Matched Layers (PMLs), allowing to consider delocalized wavefunctions, as observed when quantum relativistic particles are subject to strong fields. Thanks to a relatively new and simple Fourier-based discretization of spatial differential operators, it is possible to impose PML for solving the Dirac equation on a bounded domain. Although overall, the Fourier-based method still imposes periodic boundary conditions, the outgoing/incoming waves are in fact mainly absorbed. We think that due to the simplicity of the proposed method, most of existing Fourier-based codes could easily be modified to include the proposed methodology. The Dirac equation is a relativistic wave equation which has gain much attention these past 15 years due to the development of 2-d material, such as graphene [40, 41, 70], intense-laser-molecule interaction [38, 54, 83, 92], in particular for pair production [47, 72, 73], or from heavy ion collisions modeling and simulation for quarkantiquark production [52, 90, 106]. In the same time, there has been a tremendous progress

Email addresses: xavier antoine@univ-lorraine.fr (Xavier ANTOINE), elorin@math.carleton.ca (Emmanuel LORIN) 
in the development of efficient and accurate computational methods for the solution to the Dirac equation [6, 16, 18, 19, 24, 43, 45, 48, 49, 59, 72, 78, 83, 94], modeling in particular molecules subject to intense external laser fields. In this spirit, we have developed [47], a Schwinger-like pair production procedure from the interaction of intense laser fields with heavy molecules occuring at specific resonances [46, 47].

In this paper, we are more specificially interested in the relativistic interaction of atoms, molecules or wavepackets with intense and short laser pulses. The key point is that the laser field actually delocalizes the wavefunction, and the latter can actually interact with the domain boundary. In order to avoid artificial reflections, it is necessary to impose absorbing boundary conditions [7], or to include in the equation absorbing complex potentials or perfectly matched layers [88]. Theoretically, this approach benefits from spectral convergence (for smooth functions) and from the simplicity of Fourier-based methods, more generally pseudospectral methods, on bounded domains reducing the periodic boundary condition effect thanks to artificial wave absorption at the domain boundary. Perfectly Matched Layers are now widely used in many engineering and physics simulations codes [3, 20, 21, 28, 29, 30, 39, 66, 67, 86, 101, 102, 108, 109] to model exterior domains and to avoid unphysical reflections at the domain boundary. PML for the Dirac equation were developed in [88, and then approximated using a finite difference method. The derivation of high-order absorbing boundary conditions (ABCs) for the Dirac equation were proposed in [7]. We also refer to [8] for an overview of PMLs and ABCs for quantum wave equations including the Dirac equation and to [6, 12, 13, 16, 19, 24, 43, 48, 50, 63, 64, 74, 78, 84, for different approaches for solving the Dirac equation in real or Fourier space. We insist on the fact that the purpose of this paper is not to provide new PMLs for the Dirac equation, but rather to develop an efficient pseudospectral method for solving Dirac Initial Boundary Value Problems (IBVP).

The combination of pseudospectral methods and PMLs is possible thanks to the following simple. For example in the $x$-direction, let us denote by $\xi_{x}$ the dual Fourier variable and by $\mathcal{F}_{x}$ (resp. $\mathcal{F}_{x}^{-1}$ ) the Fourier (resp. inverse Fourier) transform in $x$. We consider $a$ as a given $x$-dependent function. Then, for any $H^{1}$-function $f$, it is possible to formally rewrite $a(x) \partial_{x} f(x)$ as $\mathcal{F}_{x}^{-1}\left(a(x)\right.$ i $\left.\xi_{x} \mathcal{F}_{x}(f)\left(\xi_{x}\right)\right)(x)$ on an unbounded domain (pseudodifferential operator representation [98]). Let us remark that the latter is a real space function, although the derivative is approximated using the Fourier transform, and the function $\left(x, \xi_{x}\right) \mapsto \mathrm{i} a(x) \xi_{x}$ is nothing but the symbol of $a(x) \partial_{x}$. In practice the function $a$ involves the stretching coordinates function modeling the PML on a bounded domain allowing real space non-reflecting conditions at the domain boundary.

The time-dependent Dirac equation under consideration reads 69]

$$
\mathrm{i} \partial_{t} \psi(t, \mathbf{x})=H \psi(t, \mathbf{x})
$$

where $\psi(t, \mathbf{x})$ is the time and coordinate dependent four-spinor, and $H$ is the Hamiltonian operator. The latter is given by

$$
H=\boldsymbol{\alpha} \cdot[c \mathbf{p}-e \mathbf{A}(t, \mathbf{x})]+\beta m c^{2}+\mathbb{I}_{4} V(t, \mathbf{x}),
$$


where the momentum operator is $\mathbf{p}=-\mathbf{i} \boldsymbol{\nabla}$. More specifically, the Dirac equation reads

$$
\begin{aligned}
\mathbf{i} \partial_{t} \psi(t, \mathbf{x})= & \left\{\alpha_{x}\left[-\mathbf{i} c \partial_{x}-e A_{x}(t, \mathbf{x})\right]\right. \\
& +\alpha_{y}\left[-\mathbf{i} c \partial_{y}-e A_{y}(t, \mathbf{x})\right] \\
& +\alpha_{z}\left[-\mathbf{i} c \partial_{z}-e A_{z}(t, \mathbf{x})\right] \\
& \left.+\beta m c^{2}+\mathbb{I}_{4} V(t, \mathbf{x})\right\} \psi(t, \mathbf{x}),
\end{aligned}
$$

where $\psi(t, \mathbf{x}) \in L^{2}\left(\mathbb{R}^{3}\right) \otimes \mathbb{C}^{4}$ is the time and coordinate $(\mathbf{x}=(x, y, z))$ dependent fourspinor. In (3), $\mathbf{A}(t, \mathbf{x})$ represents the three space components of the electromagnetic vector potential, $V(t, \mathbf{x})=e A_{0}(t, \mathbf{x})+V_{\text {nuc. }}(\mathbf{x})$ is the sum of the scalar and interaction potentials, $e$ is the electric charge (with $e=-|e|$ for an electron), $\mathbb{I}_{4}$ is the $4 \times 4$ unit matrix and $\boldsymbol{\alpha}=\left(\alpha_{\nu}\right)_{\nu=x, y, z}, \beta$ are the Dirac matrices. In this work, the Dirac representation is used, where

$$
\alpha_{\nu}=\left[\begin{array}{cc}
0 & \sigma_{\nu} \\
\sigma_{\nu} & 0
\end{array}\right] \quad, \quad \beta=\left[\begin{array}{cc}
\mathbb{I}_{2} & 0 \\
0 & -\mathbb{I}_{2}
\end{array}\right]
$$

The $\sigma_{\nu}$ are the usual $2 \times 2$ Pauli matrices defined as

$$
\sigma_{x}=\left[\begin{array}{ll}
0 & 1 \\
1 & 0
\end{array}\right] \quad, \sigma_{y}=\left[\begin{array}{cc}
0 & -i \\
i & 0
\end{array}\right] \quad \text { and } \sigma_{z}=\left[\begin{array}{cc}
1 & 0 \\
0 & -1
\end{array}\right]
$$

while $\mathbb{I}_{2}$ is the $2 \times 2$ unit matrix. Note that the light velocity $c$ and fermion mass $m$ are kept explicit in Eq. (2), allowing to adapt the method easily to natural or atomic units (a.u.).

The paper is organized as follows. We recall the basics of PMLs for the Dirac equation in Section 2. Section 3 is dedicated to the derivation of the pseudospectral approximation applied to IBVP for the time-dependent Dirac equation on a bounded domain with PML. Numerical experiments are presented in Section 4. We finally conclude in Section 5.

\section{PML for the Dirac equation}

The time-dependent Dirac equation is considered on a bounded (truncated) physical domain denoted by $\mathcal{D}_{\text {Phy }}$. We i) add a layer $\mathcal{D}_{\text {PML }}$ surrounding $\mathcal{D}_{\text {Phy }}$, and ii) stretch the coordinates in all the directions. The overall computational domain is next defined by: $\mathcal{D}=\overline{\mathcal{D}_{\text {Phy }} \cup \mathcal{D}_{\text {PML }}}$. We refer to [8] for the construction of PMLs for quantum wave equations and more specifically to [88] for the derivation and analysis of PMLs for the Dirac equation.

The starting point is the stretching of the real coordinates in the complex plane. In [88, the author uses the following variables

$$
\widetilde{\nu}=\nu+\frac{i}{\omega} \int_{L_{\nu}^{*}}^{\nu} \sigma(s) d s
$$


where $\omega$ is the dual Fourier variable to $t$ and the so-called absorbing function $\sigma$ is such that $\sigma(\nu)=0$ if $s<L_{\nu}^{*}$, for some imposed $L_{\nu}^{*}<L_{\nu}$. Then, the partial derivative $\partial_{\nu}$, with $\nu=x, y, z$, is shown to be formally transformed into

$$
\begin{aligned}
\partial_{\nu} \psi(t, \cdot) \rightarrow & \frac{\partial_{t}}{\partial_{t}+\widetilde{\sigma}_{\nu}(\nu)} \partial_{\nu} \psi(t, \cdot) \\
& =\frac{1}{2 \pi} \int_{\mathbb{R}} \frac{\omega e^{\mathrm{i} \omega t}}{\omega-\mathrm{i} \widetilde{\sigma}_{\nu}(\nu)} \partial_{\nu} \mathcal{F}_{t} \psi(\omega, \cdot) d \omega,
\end{aligned}
$$

where $\mathcal{F}_{t}\left(\right.$ resp. $\left.\mathcal{F}_{\nu}\right)$ denotes the Fourier transform with respect to $t$ (resp. $\left.\nu\right)$ and

$$
\widetilde{\sigma}_{\nu}(\nu)= \begin{cases}\sigma\left(|\nu|-L_{\nu}\right), & L_{\nu}^{*} \leqslant|\nu|<L_{\nu}, \\ 0, & |\nu|<L_{\nu}^{*} .\end{cases}
$$

Let us remark that the transformation (5) can also be formally rewritten as

$$
\partial_{\nu} \psi(t, \cdot) \rightarrow \frac{1}{1+\widetilde{\sigma}_{\nu}(\nu) \partial_{t}^{-1}} \partial_{\nu} \psi(t, \cdot) .
$$

In the present paper, we rather consider a more simple change of variables [109] involving only the space variable

$$
\widetilde{\nu}=\nu+e^{\mathrm{i} \theta} \int_{L_{\nu}^{*}}^{\nu} \sigma(s) d s,
$$

where $\theta \in(0, \pi / 2)$. We then define

$$
S_{\nu}(\nu):=1+e^{\mathrm{i} \theta_{\nu}} \widetilde{\sigma}(\nu),
$$

with $\nu=x, y, z$. In the following, the partial derivatives are simply transformed into

$$
\partial_{\nu} \rightarrow \frac{1}{S_{\nu}(\nu)} \partial_{\nu}=\frac{1}{1+e^{\mathrm{i} \theta_{\nu}} \widetilde{\sigma}_{\nu}(\nu)} \partial_{\nu},
$$

where $\widetilde{\sigma}$ vanishes in $\mathcal{D}_{\text {Phy }}$ and $S_{\nu}$ is equal to 1 . From now on, let us consider the transformation (7), and the associated new Hamiltonian

$$
H_{\mathrm{PML}}=\boldsymbol{\alpha} \cdot[c \boldsymbol{T} \cdot \mathbf{p}-e \mathbf{A}(t, \mathbf{x})]+\beta m c^{2}+\mathbb{I}_{4} V(t, \mathbf{x}),
$$

where $\boldsymbol{T}(x, y, z)=\left(T_{x}(x), T_{y}(y), T_{z}(z)\right)^{T}$ is such that

$$
T_{\nu}(\nu)=\frac{1}{S_{\nu}(\nu)} \text { with } \nu=x, y, x .
$$

Several types of functions can be selected. An exhaustive study of the absorbing functions $\sigma$ is proposed in [5] for (nonlinear) Schrödinger equations

$$
\begin{array}{ll}
\text { Type I: } \sigma_{0}\left(\nu+\delta_{\nu}\right)^{2}, & \text { Type II: } \sigma_{0}\left(\nu+\delta_{\nu}\right)^{3}, \\
\text { Type III: }-\sigma_{0} / \nu, & \text { Type IV: } \sigma_{0} / \nu^{2}, \\
\text { Type V: }-\sigma_{0} / \nu-\sigma_{0} / \delta_{\nu}, & \text { Type VI: } \sigma_{0} / \nu^{2}-\sigma_{0} / \delta_{\nu}^{2},
\end{array}
$$


for $\nu=x, y, z$ and $\delta_{\nu}=L_{\nu}-L_{\nu}^{*}>0$. The main difficulty from the pseudospectral point of view is the space-dependence of the coefficients $T_{\nu}$ which prevents the direct application of the Fourier transform on the equation. A simple trick will however allow for combining the efficiency of the pseudospectral method and the computation of the non-constant coefficient Dirac equation.

\section{PSeudospectral-PML (PS-PML) method}

A splitting of the Dirac Hamiltonian is used below. Although operator splitting is not fundamentally required in the proposed methodology, it allows to simplify the implementation of the method while keeping a good accuracy. The directional splitting provides a very efficient parallelization (per slice) of the algorithm. On the other hand, as the Dirac matrices do not commute, the splitting naturally induces errors (including possible numerical dispersion). As a consequence, the chosen splitting should have an order at least equal to the order of the time integration solvers. Based on (2), we define the operators

$$
\begin{aligned}
A & =-i c T_{x}(x) \alpha_{x} \partial_{x} \\
B & =-i c T_{y}(y) \alpha_{y} \partial_{y} \\
C & =-i c T_{z}(z) \alpha_{z} \partial_{z}, \\
D & =\beta m c^{2}+\mathbb{I}_{4} V(t, \mathbf{x})-e \boldsymbol{\alpha} \cdot \mathbf{A}(t, \mathbf{x}) .
\end{aligned}
$$

From time $t_{n}$ to $t_{n+1}$, we then successively solve [78] (the $\mathbf{x}$-dependence in the wavefunction argument is removed for notational convenience)

$$
\begin{aligned}
\mathrm{i} \partial_{t} \psi^{(1)}(t) & =A \psi^{(1)}(t), \psi^{(1)}\left(t_{n}\right)=\psi^{n}, \\
\mathrm{i} \partial_{t} \psi^{(2)}(t) & =B \psi^{(2)}(t), \psi^{(2)}\left(t_{n}\right)=\psi^{(1)}\left(t_{n_{1}}\right), t \in\left[t_{n}, t_{n_{1}}\right) \\
\left.\mathrm{i} \partial_{t} \psi_{n_{2}}\right) & (3)(t)=C \psi^{(3)}(t), \psi^{(3)}\left(t_{n}\right)=\psi^{(2)}\left(t_{n_{2}}\right), t \in\left[t_{n}, t_{n_{3}}\right) \\
\mathrm{i} \partial_{t} \psi^{(4)}(t) & =D \psi^{(4)}(t), \psi^{(4)}\left(t_{n}\right)=\psi^{(3)}\left(t_{n_{3}}\right), t \in\left[t_{n}, t_{n+1}\right) \\
\text { and } \psi^{n+1} & =\psi^{(4)}\left(t_{n+1}\right),
\end{aligned}
$$

where $t_{n_{i}}-t_{n}=\Delta t$ for $i \in\{1, \cdots, 3\}$. In the next subsections, we detail the space-time

approximations of equations (14)-(17). Cylindrical coordinates could be used as well (see e.g. [48]).

\subsection{Pseudospectral approximation in space}

We consider for convenience the 3-dimensional system in cartesian coordinates, for $\nu=$ $x, y, z$ in the domain $\mathcal{D}=\left[-a_{x}, a_{x},\right] \times\left[-a_{y}, a_{y}\right] \times\left[-a_{z}, a_{z}\right]$,

$$
\mathrm{i} \partial_{t} \psi(t)=-\mathrm{i} c \alpha_{\nu} T_{\nu}(\nu) \partial_{\nu} \psi(t), \psi\left(t_{n}\right)=\psi^{n}, \quad t \in\left[t_{n}, t_{n+1}\right)
$$

and we use the same notations as in [4]. We first diagonalize $\alpha_{\nu}=\Pi_{\nu} \Lambda \Pi_{\nu}^{\dagger}$, where

$$
\Lambda=\left(\begin{array}{cccc}
1 & 0 & 0 & 0 \\
0 & 1 & 0 & 0 \\
0 & 0 & -1 & 0 \\
0 & 0 & 0 & -1
\end{array}\right)
$$


and $\Pi_{\nu}^{\dagger}=\bar{\Pi}_{\nu}^{T}$. The matrices $\Pi_{\nu}$ are defined as follows

$$
\begin{gathered}
\Pi_{x}=\frac{1}{\sqrt{2}}\left(\begin{array}{cccc}
0 & 1 & 1 & 0 \\
1 & 0 & 0 & -1 \\
1 & 0 & 0 & 1 \\
0 & 1 & -1 & 0
\end{array}\right), \\
\Pi_{y}=\frac{1}{\sqrt{2}}\left(\begin{array}{cccc}
0 & -i & -i & 0 \\
1 & 0 & 0 & 1 \\
-i & 0 & 0 & i \\
0 & 1 & -1 & 0
\end{array}\right), \\
\Pi_{z}=\frac{1}{\sqrt{2}}\left(\begin{array}{cccc}
1 & 0 & 0 & -1 \\
0 & -1 & -1 & 0 \\
1 & 0 & 0 & 1 \\
0 & 1 & -1 & 0
\end{array}\right) .
\end{gathered}
$$

We set $\phi:=\Pi_{\nu}^{\dagger} \psi$, which then satisfies

$$
\begin{aligned}
\mathrm{i} \partial_{t} \phi(t) & =-\mathrm{i} c \Lambda T_{\nu}(\nu) \partial_{\nu} \phi(t), \\
\phi\left(t_{n}\right) & =\Pi_{\nu}^{\dagger} \psi^{n}, \quad t \in\left[t_{n}, t_{n+1}\right) .
\end{aligned}
$$

We denote the set of grid-points by

$$
\mathcal{D}_{N_{x}, N_{y}, N_{z}}=\left\{\mathbf{x}_{k_{1}, k_{2}, k_{3}}=\left(x_{k_{1}}, y_{k_{2}}, z_{k_{3}}\right)\right\}_{\left(k_{1}, k_{2}, k_{3}\right) \in \mathcal{O}_{N_{x} N_{y} N_{z}}}
$$

with

$$
\mathcal{O}_{N_{x} N_{y} N_{z}}=\left\{k_{1}=0, \cdots, N_{x}-1 ; k_{2}=0, \cdots, N_{y}-1 ; k_{3}=0, \cdots, N_{z}-1\right\} .
$$

Then, let us introduce the following mesh sizes

$$
\begin{aligned}
& x_{k_{1}+1}-x_{k_{1}}=h_{x}=2 a_{x} / N_{x} \\
& y_{k_{2}+1}-y_{k_{2}}=h_{y}=2 a_{y} / N_{y} \\
& z_{k_{3}+1}-z_{k_{3}}=h_{z}=2 a_{z} / N_{z} .
\end{aligned}
$$

The corresponding discrete wavenumbers are defined by $\boldsymbol{\xi}_{p, q, r}:=\left(\xi_{p}, \xi_{q}, \xi_{r}\right)$, where $\xi_{p}=$ $p \pi / a_{x}$ with $p \in\left\{-N_{x} / 2, \cdots, N_{x} / 2-1\right\}, \xi_{q}=q \pi / a_{y}$ with $q \in\left\{-N_{y} / 2, \cdots, N_{y} / 2-1\right\}$ and $\xi_{r}=r \pi / a_{z}$ with $r \in\left\{-N_{z} / 2, \cdots, N_{z} / 2-1\right\}$. In the sequel of the paper, we denote by $\phi^{(\ell)}$, with $\ell \in\{1,2,3,4\}$, the $\ell$ th component of the spectral approximation of $\phi=\Pi_{\nu}^{\dagger} \psi$. We also use the notation $\phi_{k_{1}}^{(\ell)}(t, y, z)=\phi^{(\ell)}\left(t, x_{k_{1}}, y, z\right), \phi_{k_{2}}^{(\ell)}(t, x, z)=\phi^{(\ell)}\left(t, x, y_{k_{2}}, z\right)$ and 
$\phi_{k_{3}}^{(\ell)}(t, x, y)=\widetilde{\phi}^{(\ell)}\left(t, x, y, z_{k_{3}}\right)$. The partial Fourier coefficients are such that

$$
\left\{\begin{array}{l}
\widehat{\phi}_{p}^{(\ell)}(t, y, z)=\sum_{k_{1}=0}^{N_{x}-1} \phi_{k_{1}}^{(\ell)}(t, y, z) e^{-\mathrm{i} \xi_{p}\left(x_{k_{1}}+a_{x}\right)}, \\
\widehat{\phi}_{q}^{(\ell)}(t, x, z)=\sum_{k_{2}=0}^{N_{y}-1} \phi_{k_{2}}^{(\ell)}(t, x, z) e^{-\mathrm{i} \xi_{q}\left(y_{k_{2}}+a_{y}\right)}, \\
\widehat{\phi}_{r}^{(\ell)}(t, x, y)=\sum_{k_{3}=0}^{N_{z}-1} \phi_{k_{3}}^{(\ell)}(t, x, y) e^{-\mathrm{i} \xi_{r}\left(z_{k_{3}}+a_{z}\right)} .
\end{array}\right.
$$

We can then introduce the inverse partial Fourier pseudospectral approximations, in the $x-$, $y$ - and $z$-directions, respectively,

$$
\left\{\begin{aligned}
\widetilde{\phi}_{k_{1}}^{(\ell)}(t, y, z) & =\frac{1}{N_{x}} \sum_{p=-N_{x} / 2}^{N_{x} / 2-1} \widehat{\phi}_{p}^{(\ell)}(t, y, z) e^{\mathrm{i} \xi_{p}\left(x_{k_{1}}+a_{x}\right)} \\
\widetilde{\phi}_{k_{2}}^{(\ell)}(t, x, z) & =\frac{1}{N_{y}} \sum_{q=-N_{y} / 2}^{N_{y} / 2-1} \widehat{\phi}_{q}^{(\ell)}(t, x, z) e^{\mathrm{i} \xi_{q}\left(y_{k_{2}}+a_{y}\right)} \\
\widetilde{\phi}_{k_{3}}^{(\ell)}(t, x, y) & =\frac{1}{N_{z}} \sum_{r=-N_{z} / 2}^{N_{z} / 2-1} \widehat{\phi}_{r}^{(\ell)}(t, x, y) e^{\mathrm{i} \xi_{r}\left(z_{k_{3}}+a_{z}\right)}
\end{aligned}\right.
$$

We then denote

$$
\widetilde{\phi}:=\left\{\widetilde{\phi}^{\ell}\right\}_{1 \leqslant \ell \leqslant 4},
$$

and define the approximate first-order partial derivatives such that for any $\nu$

$$
\partial_{\nu} \phi^{(\ell)}\left(t_{n}, \mathbf{x}_{k_{1}, k_{2}, k_{3}}\right) \approx\left\{\left[\left[\partial_{\nu}\right]\right] \phi^{(\ell)}\right\}_{k_{1}, k_{2}, k_{3}}
$$

and

$$
\begin{aligned}
&\left\{\left[\left[\partial_{x}\right]\right] \phi^{(\ell)}\right\}_{k_{1}, k_{2}, k_{3}}:=\frac{1}{N_{x}} \sum_{p=-N_{x} / 2}^{N_{x} / 2-1} \mathrm{i} \xi_{p}\left(\widehat{\widetilde{\phi}}_{k_{2}, k_{3}}\right)_{p} e^{\mathrm{i} \xi_{p}\left(x_{k_{1}}+a_{x}\right)}, \\
&\left\{\left[\left[\partial_{y}\right]\right] \phi^{(\ell)}\right\}_{k_{1}, k_{2}, k_{3}}:=\frac{1}{N_{y}} \sum_{q=-N_{y} / 2}^{N_{y} / 2-1} \mathrm{i} \xi_{q}\left(\widehat{\widetilde{\phi}}_{k_{1}, k_{3}}^{(\ell)}\right)_{q} e^{\mathrm{i} \xi_{q}\left(x_{k_{2}}+a_{y}\right)}, \\
&\left\{\left[\left[\partial_{z}\right]\right] \phi^{(\ell)}\right\}_{k_{1}, k_{2}, k_{3}}:=\frac{1}{N_{z}} \sum_{r=-N_{z} / 2}^{N_{z} / 2-1} \mathrm{i} \xi_{r}\left(\widehat{\widetilde{\phi}}_{k_{1}, k_{2}}^{(\ell)}\right)_{r} e^{\mathrm{i} \xi_{r}\left(x_{k_{3}}+a_{z}\right)} .
\end{aligned}
$$

In the following, the index $h$ will be used (e.g. in $\phi_{h}^{n}=\left\{\phi_{k_{1}, k_{2}, k_{3}}^{n}\right\}_{k_{1}, k_{2}, k_{3}}$ ) to denote a spectral approximation to a given wavefunction $\left(\right.$ e.g. $\left.\phi^{n}\right)$. This discretization not only allows to select the spatial step as large as wanted, but it also preserves the very high spatial accuracy, the parallel computing structure and the scalability of the split method developed in [43]. 


\subsection{Time-Splitting PSeudospectral method with PML (TSSP-PML)}

As proposed before, we use a splitting of the Time-Dependent Dirac Equation into four time-dependent systems (10), (11), (12) and (13). We also define $\mathbf{x}_{h}=\left(x_{h}, y_{h}, z_{h}\right)$ as the nodes of a real-space grid. At iteration time $n$, the approximate wave function is denoted by $\psi_{h}^{n}$. From a stability point of view, an implicit scheme should be implemented. The TSSP (Time-Splitting pseudoSPectral) algorithm then reads

1. First step: integration of the generalized transport equation in Fourier space in the $x$-direction. One sets $\phi_{h}^{n}:=\prod_{x}^{\dagger} \psi_{h}^{n}$, and the system

$$
\partial_{t} \phi+c T_{x}(x) \Lambda \partial_{x} \phi=0, \quad \phi\left(t_{n}, \cdot\right)=\Pi_{x}^{\dagger} \psi\left(t_{n}, \cdot\right),
$$

is approximately solved by

$$
\phi_{h}^{n_{1}}=\phi_{h}^{n}-c \Delta t T_{x}\left(x_{h}\right) \Lambda\left[\left[\partial_{x}\right]\right] \phi_{h}^{n_{1}},
$$

where $[[\cdot]]$ is defined in (24). We then deduce $\psi_{h}^{n_{1}}=\Pi_{x} \phi_{h}^{n_{1}}$. This gives an approximation of Eq. (14), where the corresponding operator is denoted by $\mathcal{P}_{h}^{(x)}(\Delta t)$.

2. Second step: integration of the generalized transport equation in Fourier space in the $y$-direction

$$
\partial_{t} \phi+c T_{y}(y) \Lambda \partial_{y} \phi=0
$$

We set $\chi_{h}^{n_{1}}:=\Pi_{y}^{\dagger} \psi_{h}^{n_{1}}$ and we approximately solve (15) by using the following scheme

$$
\left.\phi_{h}^{n_{2}}=\chi_{h}^{n_{1}}-c \Delta t T_{y}^{(} y_{h}\right) \Lambda\left[\left[\partial_{y}\right]\right] \chi_{h}^{n_{2}} .
$$

We then obtain $\psi_{h}^{n_{2}}=\Pi_{y} \phi_{h}^{n_{2}}$.

3. Third step: setting $\chi_{h}^{n_{2}}:=\prod_{z}^{\dagger} \psi_{h}^{n_{2}}$, we apply the same procedure in the $z$-direction, and compute $\psi_{h}^{n_{3}}$. The corresponding operator is denoted by $\mathcal{P}_{h}^{(z)}(\Delta t)$.

4. Fourth step: integration of the source from $t_{n}$ to $t_{n+1}$

$$
\begin{aligned}
\psi_{h}^{n+1}= & T \exp \left[-\mathrm{i} \int_{t_{n}}^{t_{n+1}} d \tau\left[\beta m c^{2}-e \boldsymbol{\alpha} \cdot \mathbf{A}_{h}(\tau)\right]\right] \\
& \times \exp \left[-\mathrm{i} e \int_{t_{n}}^{t_{n+1}} d \tau V_{h}(\tau)\right] \psi_{h}^{n_{3}}
\end{aligned}
$$

where $\mathbf{A}_{h}$ is the approximate electric potential. The corresponding operator is denoted by $\mathcal{Q}_{h}^{n}(\Delta t)$.

This splitting scheme is implicit, and can be compactly expressed as a composition of the four operators

$$
\psi_{h}^{n+1}=\mathcal{Q}_{h}^{n}(\Delta t) \mathcal{P}_{h}^{(z)}(\Delta t) \mathcal{P}_{h}^{(y)}(\Delta t) \mathcal{P}_{h}^{(x)}(\Delta t) \psi_{h}^{n}
$$


In practice, a second-order splitting scheme should be implemented, such as

$$
\begin{aligned}
\psi_{h}^{n+1}= & \mathcal{P}_{h}^{(x)}\left(\frac{\Delta t}{8}\right) \mathcal{P}_{h}^{(y)}\left(\frac{\Delta t}{4}\right) \mathcal{P}_{h}^{(x)}\left(\frac{\Delta t}{8}\right) \mathcal{P}_{h}^{(z)}\left(\frac{\Delta t}{2}\right) \mathcal{P}_{h}^{(x)}\left(\frac{\Delta t}{8}\right) \mathcal{P}_{h}^{(y)}\left(\frac{\Delta t}{4}\right) \mathcal{P}_{h}^{(x)}\left(\frac{\Delta t}{8}\right) \mathcal{Q}_{h}^{n}(\Delta t) \\
& \mathcal{P}_{h}^{(x)}\left(\frac{\Delta t}{8}\right) \mathcal{P}_{h}^{(y)}\left(\frac{\Delta t}{4}\right) \mathcal{P}_{h}^{(x)}\left(\frac{\Delta t}{8}\right) \mathcal{P}_{h}^{(z)}\left(\frac{\Delta t}{2}\right) \mathcal{P}_{h}^{(x)}\left(\frac{\Delta t}{8}\right) \mathcal{P}_{h}^{(y)}\left(\frac{\Delta t}{4}\right) \mathcal{P}_{h}^{(x)}\left(\frac{\Delta t}{8}\right) \psi_{h}^{n}
\end{aligned}
$$

Thanks to the operator $\left[\left[\partial_{\nu}\right]\right]$, it is possible to implement the real-space approximation of the overall wave function on a bounded domain imposing in particular PMLs, resulting in the TSSP-PML scheme.

\subsection{Mathematical analysis}

In this section, we discuss the stability and convergence of the pseudospectral method developed in Subsection 3.2. The proof mainly relies on [15, 55]. To simplify the presentation, we assume that the computational domain is $\mathcal{D}=(\mathbb{T}(0,2 \pi))^{3}$. The convergence analysis contains two main parts. We first consider the one-dimensional case for $\phi_{0}=\phi(0, \cdot) \in$ $\left(H^{s}(\mathbb{T}(0,2 \pi))\right)^{4}$ such that $\Lambda=\operatorname{diag}(1,1,-1,-1) \in \mathbb{R}^{4 \times 4}$, and

$$
\partial_{t} \phi(t, x)+c T(x) \Lambda \phi(t, x)=0, \quad x \in \mathbb{T}(0,2 \pi), T_{x} \in C^{1}[0,2 \pi],
$$

where $T_{x}(x)=1 / S_{x}(x)$ is the inverse of an absorbing function $S_{x}$ as defined in (6), which is assumed to be differentiable with bounded derivative. Using the same notations as [15, we here define

$$
P_{N}[\phi]:=\sum_{|k| \leqslant N}\left(\frac{1}{2 \pi} \int_{\mathbb{T}} \phi(x) e^{-i k x} d x\right) e^{i k x}, \quad \Psi_{N}[\phi]=\widetilde{\phi}
$$

where $\widetilde{\phi}$ is given by 23. Thus, we have $P_{N}[\phi]=\Psi_{N}[\phi]+A_{N}[\phi]$, where the aliasing term

$$
A_{N}[\phi](x)=\sum_{|k| \leqslant N}\left(\sum_{|l| \geqslant 1} \frac{1}{2 \pi} \int_{\mathbb{T}} \phi(x) e^{-i(k+N l) x} d x\right) e^{i k x}
$$

which is responsible for a loss of stability/accuracy is such that [55]

$$
\left\|A_{N}[\phi]\right\|_{\left(H^{s}\right)^{4}} \lesssim N^{s-r}\|\phi\|_{\left(H^{r}\right)^{4}}, \quad r>s>1 / 2 .
$$

This inequality illustrates that the loss of accuracy is directly related to the smoothness. Denoting $\phi_{N}(x, t)=\sum_{|k| \leqslant N} \widehat{u}_{k}(t) e^{\mathrm{i} k x}$, from 29 the scheme can be rewritten as

$$
\partial_{t} \phi_{N}(x, t)=-c T_{x}(x) \Lambda\left[\left[\partial_{x}\right]\right] \phi_{N}(x, t)=-c T_{x}(x) \Lambda \psi_{N}\left[\partial_{x} \widehat{\phi}\right]=-c T_{x}(x) \Lambda \partial_{x} \psi_{N}[\widehat{\phi}] .
$$

Let us remark that we can obtain the first step of the scheme in Subsection 3.2 as

$$
\partial_{t} \phi_{N}(x, t)+c T_{x}(x) \Lambda \partial_{x} P_{N}\left[\phi_{N}\right]=0
$$


The stability analysis then reads as follows. First, we have

$$
\left\|\phi_{N}(\cdot, t)\right\|_{\left(L^{2}\right)^{4}}^{2}=-2 \Lambda \int c T_{x}(x) \phi_{N}^{\dagger}(x, t) \partial_{x} P_{N}\left[\phi_{N}\right] d x-2 \Lambda \int c T_{x}(x) \phi_{N} \partial_{x} A_{N}\left[\phi_{N}\right] d x
$$

with

$$
2 \Lambda \int c T_{x}(x) \phi_{N}^{\dagger}(x, t) \partial_{x} P_{N}\left[\phi_{N}\right] d x \leqslant c\left\|T_{x}^{\prime}\right\|_{\infty}\left\|\phi_{N}\right\|_{\left(L^{2}\right)^{4}}^{2}
$$

Finally, according to [15, 55, we conclude that there exists a constant $K_{s}>0$ such that

$$
\left\|\phi_{N}(\cdot, t)\right\|_{\left(L^{2}\right)^{4}} \lesssim N e^{c K_{s}\left\|T_{x}^{\prime}\right\|_{L^{\infty} t}}\left\|\phi_{N}(\cdot, 0)\right\|_{\left(L^{2}\right)^{4}} .
$$

We then have

Lemma 3.1. For $s>2$, there exists a constant $K_{s}>0$ such that:

$$
\left\|\phi_{N}(\cdot, t)-\Psi_{N}[\phi]\right\|_{\left(L^{2}\right)^{4}} \lesssim e^{c K_{s}\left\|T_{x}^{\prime}\right\|_{L^{\infty} t}}\left(N^{1-s}\|\phi(\cdot, 0)\|_{\left(H^{s}\right)^{4}}+N^{2-s} \max \|\phi(\cdot, \tau)\|_{\left(H^{s}\right)^{4}}\right)
$$

We now consider the three-dimensional split system. For any transition matrix $\Pi_{\nu}$ defined in $190,\left\|\Pi_{\nu}^{\dagger} \psi\right\|=\|\phi\|$, the above estimate (27) is valid for the first to third steps of the scheme 3.2. Finally, the contribution of the fourth step 25 is straightforward in the error estimate. We then have the following proposition.

Proposition 3.1. Let us assume that $\psi_{0}=\psi(0, \cdot) \in\left(H^{s}(\mathbb{T}(0,2 \pi))\right)^{4}$ and that for $\nu=$ $x, y, z, T_{\nu}:=1 / S_{\nu} \in W^{1, \infty}(0,2 \pi)$. Then, the numerical scheme derived in Subsection (3.2) is such that

$$
\begin{aligned}
\left\|\psi_{N}(\cdot, t)-\Psi_{N}[\psi]\right\|_{\left(L^{2}\right)^{4}} \lesssim & \exp \left(\left(c K_{s}\left\|T_{x}^{\prime}\right\|_{L^{\infty}}+\|\mathbf{A}\|_{\infty}\right) t\right) \\
& \times\left(N^{1-s}\|\psi(\cdot, 0)\|_{\left(H^{s}\right)^{4}}+N^{2-s} \max \|\psi(\cdot, \tau)\|_{\left(H^{s}\right)^{4}}\right),
\end{aligned}
$$

for some constant $K_{s}>0$.

This proposition shows the spectral convergence of the derived pseudospectral method, in the sense that the convergence increases with the order of regularity of the solution. It also precises the dependence on the regularity of the absorption functions $S_{\nu}$, and more specifically the regularity of $T_{\nu}$ but also on ii) $\left\|T_{\nu}^{\prime}\right\|_{L^{\infty}}$. Assuming that $T_{\nu}$ is smooth, we get

$$
\frac{d}{d \nu}\left(\frac{1}{S_{\nu}(\nu)}\right)=-\frac{S_{\nu}^{\prime}(\nu)}{S_{\nu}^{2}(\nu)}=-\frac{e^{\mathrm{i} \theta} \widetilde{\sigma}_{\nu}^{\prime}(\nu)}{\left(1+e^{\mathrm{i} \theta} \widetilde{\sigma}_{\nu}(\nu)\right)^{2}}
$$

where $\widetilde{\sigma}$ is differentiable or piecewise differentiable. More specifically,

- for the Type-I PML, $\widetilde{\sigma}_{\nu}$ is differentiable

$$
\widetilde{\sigma}_{\nu}^{\prime}(\nu)= \begin{cases}2 \operatorname{sgn}(\nu) \sigma_{0}\left(|\nu|-L_{\nu}+\delta_{\nu}\right), & L_{\nu}^{*} \leqslant|\nu|<L_{\nu}, \\ 0, & |\nu|<L_{\nu}^{*},\end{cases}
$$

where $\delta_{\nu}=L_{\nu}-L_{\nu}^{*}$. Thus, we obtain that

$$
\left|\frac{d}{d \nu}\left(\frac{1}{S_{\nu}(\nu)}\right)\right|=\frac{2 \sigma_{0}\left(L_{\nu}-|\nu|\right)}{\left|1+e^{\mathrm{i} \theta} \widetilde{\sigma}_{\nu}(\nu)\right|^{2}} \leqslant 2 \sigma_{0} \delta_{\nu} .
$$


- for the Type-II PML, $\widetilde{\sigma}_{\nu}$ is also differentiable and

$$
\left|\frac{d}{d \nu}\left(\frac{1}{S_{\nu}(\nu)}\right)\right| \leqslant 3 \delta_{\nu}^{2} .
$$

- for the Type-IV/VI PML, $\widetilde{\sigma}_{\nu}$ is only piecewise differentiable

$$
\widetilde{\sigma}_{\nu}^{\prime}(\nu)= \begin{cases}-2 \operatorname{sgn}(\nu) \frac{\sigma_{0}}{\left(|\nu|-L_{\nu}\right)^{3}}, & L_{\nu}^{*}<|\nu|<L_{\nu}, \\ 0, & |\nu|<L_{\nu}^{*} .\end{cases}
$$

Therefore, for $L_{\nu}^{*}<|\nu|<L_{\nu}$, one gets

$$
\left|\frac{d}{d \nu}\left(\frac{1}{S_{\nu}(\nu)}\right)\right|=\frac{2 \sigma_{0}}{\left(L_{\nu}-|\nu|\right)^{3}\left|1+e^{\mathrm{i} \theta} \widetilde{\sigma}_{\nu}(\nu)\right|^{2}} .
$$

Let us note that for $|\nu|$ close to $L_{\nu}$ we have

$$
\left|\frac{d}{d \nu}\left(\frac{1}{S_{\nu}(\nu)}\right)\right| \lesssim 2 \sigma_{0}\left(L_{\nu}-|\nu|\right)
$$

while for $|\nu|$ close to $L_{\nu}^{*}$ one gets

$$
\left|\frac{d}{d \nu}\left(\frac{1}{S_{\nu}(\nu)}\right)\right| \lesssim 2 \sigma_{0}\left(L_{\nu}-L_{\nu}^{*}\right) .
$$

For the Type-IV-VI (as well as Type-III-V) PML, the piecewise differentiability can play a role in the loss of precision of the overall method (see inequality (28)). However, this was not numerically observed in our tests. In [15], the authors propose to perform a de-aliasing thanks to a $2 / 3$-smoothing method by using the factor function $\sigma$ (see 2.12a in [15]). More precisely, for one-dimensional evolution equations

$$
\partial_{t} \phi_{N}(x, t)=-c T_{x}(x) \Lambda\left[\left[\partial_{x}\right]\right] \mathcal{S} \phi_{N}(x, t),
$$

where $\mathcal{S} \phi_{N}=\sum_{|k| \leqslant 2 N / 3} \sigma_{k} \widehat{u}_{k}(t) e^{\mathrm{i} k x}$, we expect that, for some $s>1$,

$$
\left\|\phi_{N}(\cdot, t)-\phi\right\|_{\left(L_{S}^{2}\right)^{4}} \lesssim e^{K_{s}\left\|T_{x}^{\prime}\right\|_{L^{\infty}} t}\left(N^{-s}\|\phi(\cdot, 0)\|_{\left(H^{s}\right)^{4}}+N^{2-s} \max \|\phi(\cdot, \tau)\|_{\left(H^{s}\right)^{4}}\right) .
$$

We have proposed an implicit scheme to solve the first 3 steps to Subsection 3.2, and which is based on the solution to systems of the form:

$$
\phi_{N}^{n+1}(x, t)=\phi_{N}^{n}(x, t)-c T_{x}(x) \Lambda \partial_{x} \Psi_{N}\left[\widehat{\phi}^{n+1}\right] .
$$

In the following, we also propose an alternative scheme which avoids the numerical computation to linear systems due to the implicit time-discretization. For example, for the First step, we can simply instead solve

$$
\partial_{t} \phi+c T_{x}(x) \Lambda \partial_{x} \phi=0, \phi\left(t_{n}, \cdot\right)=\Pi_{x}^{\dagger} \psi\left(t_{n}, \cdot\right),
$$


by using

$$
\phi_{h}^{n_{1}}:=\frac{1}{S_{x}\left(x_{h}\right)} \psi_{h}^{n_{1}}+\left(1-\frac{1}{S_{x}\left(x_{h}\right)}\right) \phi_{h}^{n}
$$

where $\psi_{h}^{n_{1}}$ is solution to

$$
\psi_{h}^{n_{1}}=\phi_{h}^{n}-c \Delta t \Lambda\left[\left[\partial_{x}\right]\right] \phi_{h}^{n}
$$

In other words, $\phi_{h}^{n_{1}}$ is an approximation to

$$
\phi\left(t_{n_{1}}, x\right)=\frac{1}{S_{x}(x)} \mathcal{F}_{x}^{-1}\left(\left(1-\mathrm{i} c \Delta t \xi_{x}\right) \mathcal{F}_{x}(\phi)\left(t_{n_{1}}, \xi_{x}\right)\right)+\left(1-\frac{1}{S_{x}(x)}\right) \phi\left(t_{n}, x\right) .
$$

This approximation is justified as follows. From time $t_{n}$ to $t_{n_{1}}$, we integrate 29 and get

$$
\begin{aligned}
\phi\left(t_{n_{1}}, x\right) & =\phi\left(t_{n}, x\right)-\frac{c \Delta t}{S_{x}(x)} \mathcal{F}_{x}^{-1}\left(\mathrm{i} \xi_{x} \mathcal{F}_{x}(\phi)\left(t_{n}, \xi\right)\right)+O\left(\Delta t^{2}\right) \\
& =\frac{1}{S_{x}(x)} \mathcal{F}_{x}^{-1}\left(\left(1-\mathrm{i} c \Delta t \xi_{x}\right) \mathcal{F}_{x}(\phi)\left(t_{n}, \xi\right)\right)+\left(1-\frac{1}{S_{x}(x)}\right) \phi\left(t_{n}, x\right)+O\left(\Delta t^{2}\right) .
\end{aligned}
$$

As a consequence, we trivially have

Proposition 3.2. The approximation (30)-31) is consistent with 29.

\section{Numerical simulations}

We propose some numerical experiments in 2-d, illustrating the efficiency of the combined PML-TSSP method with a second-order operator splitting. We are mainly interested in the evolution of wavepacket either subject to a static potential or to an external dynamic field. In our simulations, we have used several libraries which are listed below and consider the atomic units. We compare the effects of different absorption functions with various parameters. We can however not conclude regarding the optimal choice, although Type-IV functions are often among the most efficient. Let us note however that from a theoretical point of view (see Subsection 3.3 the accuracy of the pseudospectral method depends on the regularity of the absorption functions $S_{\nu}$, and more specifically the regularity of $1 / S_{\nu}$.

\subsection{Technical details about the code and parallel computing aspects}

The Fourier transforms are performed with the sequential and parallel version 3.3 .4 of fftw. Standard linear algebra libraries, gsl (version 1.9) and blas were also used. Finally openMPI 1.6.3 was used for message passing with non-blocking communication. The code was implemented in $\mathrm{C}++$ and the compiler is the version 4.7 .0 of gcc. The tests are performed by using a C++-code with MPI-library. The method is implemented on the cluser mammouth-parallel II from the RQCHP. The total processing power of this machine is 
333400 GFlops, and possesses 39648 cores: 3216 processors AMD Opteron 12 cores at 2.1 $\mathrm{GHz}$, and 88 processors AMD Opteron 12 cores $2.2 \mathrm{GHz}$. The total memory is $57.6 \mathrm{~TB}$ and the computer-networking communications is Infiniband QDR (4 GB/sec). Regarding the communication between processes, by default mammouth II will select the closest processors in the same node, then the processors from the nodes in the same topological ring, and finally through different switch-levels. Notice that for a low number of processors, the communication between the processes within the same node is done thanks to a shared memory. The pseudospectral method for solving the Dirac equation solver without PML is detailed in [6], and in Section 4. Let us recall the main features of the code in 2-d. In the $(x, z)$ coordinates, we proceed by alternating the directions. We first decompose the domain by layer (slice) in the $z$-direction, then

- we successively perform the evolution (FFT) sequentially in the $x$-direction, and by layer in the $z$-direction. Each processor manages one layer in $z$. A perfect scaling for this step is expected as it does not require any transmission between nodes.

- for all $x$, we perform the evolution in the $z$-direction using the parallel FFT (fftw). The performance of this step is then fully dependent on the parallelization of the one-dimensional FFT.

Notice that the presence of the PML does not deteriorate the efficiency of the overall method compared to usual FFT-methods. In particular, the computational complexity is simple to established, and is given by $C$ after $N_{T}$ time iterations

$$
C=\mathcal{O}\left(N_{T_{f}} N_{x} N_{z} \log \left(N_{x} N_{z}\right)\right)
$$

The scalability and performance of the TSSP method without PML is fully studied in [43], where in particular it is shown a good speed-up of this method.

\subsection{Numerical experiments}

The second-order TSSP-PML method (26) is implemented, where the discrete operators [[.]] given by (24) are used in the directions $\nu=x, z$. We then choose $S_{\nu}$ as follows, for $\nu=x, z$

$$
S_{\nu}(\nu)=\left\{\begin{array}{cc}
1, & |\nu|<L_{\nu}^{*}, \\
1+e^{\mathrm{i} \theta} \sigma\left(|\nu|-L_{v}\right), & L_{\nu}^{*} \leqslant|\nu|<L_{\nu} .
\end{array}\right.
$$

Test 1 : wavepacket subject to an external laser field. In this first test, we consider a wavepacket

$$
\boldsymbol{\psi}(x, y, z, 0)=\mathcal{N}[1,0,0,0]^{T} \exp \left(-\left(x^{2}+y^{2}+z^{2}\right) / \Delta^{2}\right) \times \exp \left(i k_{x} x\right),
$$

subject to an external potential and propagating with a fixed wavenumber $\boldsymbol{k}=(5,0)$, and $\Delta=128$ in (33). The time step is given by $\Delta t=4.56 \times 10^{-4} a . u$. The physical domain is $\mathcal{D}=[-8,8]^{2}$ and is discretized with $N_{x} \times N_{z}=256^{2}$ grid points. We impose a linearly 

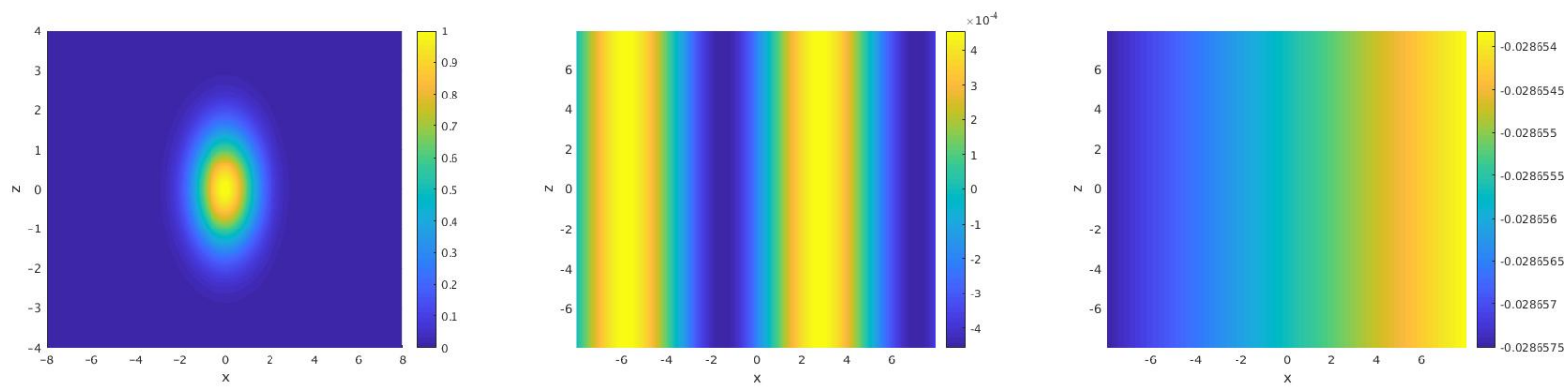

Figure 1: Test 1 : initial density (Left), $A_{z}(T, x, y)$ at times $t=0.456 a . u$. (Middle) and 1.824a.u. (Right).

polarized electric field such that $A_{x}$ is identically null, and $A_{z}(t, x, z)$ is as in (35), with $A_{0}=100 / 137, \omega=100, n=1, n^{\prime}=19$ and $T_{f}=1.824 a . u$. As an example, we report in Fig. 1 the electric potential $A_{z}$ at time $T=0.456 a . u$. and 1.824a.u. and the initial data. In Figs. 22, we compare the evolution of the density $d(T, x, z)=\sum_{i=1}^{4}\left|\psi_{i}(T, x, z)\right|^{2}$ as a function of time, with i) periodic boundary conditions with PML of ii) type III and iii) type IV, with $\sigma_{0}=10^{-2}, \theta=\pi / 4$ as well as iv) type $\mathrm{V}$ and VI with $\delta_{\nu}=L_{\nu}-L_{\nu}^{*}$. The snapshots correspond to times $t=0.456 a . u$., 1.14a.u., 1.368a.u. and $T=1.824 a . u$., for respectively 1000, 2500, 3000 and 4000 time iterations. The best absorption is obtained with the Type-VI absorbing function, although the results are relatively close with all the absorbing functions. We report in Fig. 3, the maximum of the density (in logscale) as a function of the time iterations for 6 different absorbing functions, showing that they all provide relatively good properties for truncating the computational domain.

Test 2 : 3-nucleus system. In this test, a Gaussian wavepacket centered at $(0,0)$ Fig. 4 (Left), is injected in a 3 -nucleus interaction potential Fig 4 (Middle)

$$
\begin{aligned}
V(x, z)= & -\frac{Z_{\mathrm{A}}}{\sqrt{\left(x-x_{\mathrm{A}}\right)^{2}+\left(z-z_{\mathrm{A}}\right)^{2}+2}} \\
& -\frac{Z_{\mathrm{B}}}{\sqrt{\left(x-x_{\mathrm{B}}\right)^{2}+\left(z-z_{\mathrm{B}}\right)^{2}+2}} \\
& -\frac{Z_{\mathrm{C}}}{\sqrt{\left(x-x_{\mathrm{C}}\right)^{2}+\left(z-z_{\mathrm{C}}\right)^{2}+2}}
\end{aligned}
$$

with $Z_{\mathrm{A}}=Z_{\mathrm{B}}=Z_{\mathrm{C}}=10$ a.u., $\left(x_{\mathrm{A}}, z_{\mathrm{A}}\right)=(-2,0),\left(x_{\mathrm{B}}, z_{\mathrm{B}}\right)=(2,0)$ and $\left(x_{\mathrm{C}}, z_{\mathrm{C}}\right)=(0,-2)$. The overall computational domain is $\mathcal{D}=[-6,6]^{2}$, with $N_{x} \times N_{y}=128^{2}$ grid points and $\Delta t=3 \times 10^{-3} a . u$. Notice that this time step does not allow for the capture of very small time scale effects, such as the zitterbewegung [99]. As we are here interested in larger scales effects, more specifically the absorption layer effect, we allow a larger time step. For practical application, we however suggest to use, as usual, a time step $\leqslant 1 / 2 m c^{2}\left(\approx 2.67 \times 10^{-5}\right)$ to get a better precision. We report on Fig. 4 (Right) the total density at time $T=3 \times 10^{-1}$ a.u, i.e. $d(T, x, z)$, for $(x, z) \in \mathcal{D}$, where $d(T, x, z)=\sum_{i=1}^{4}\left|\psi_{i}(T, x, z)\right|^{2}$, setting $\boldsymbol{\psi}=\left(\psi_{1}, \psi_{2}, \psi_{3}, \psi_{4}\right)$ 

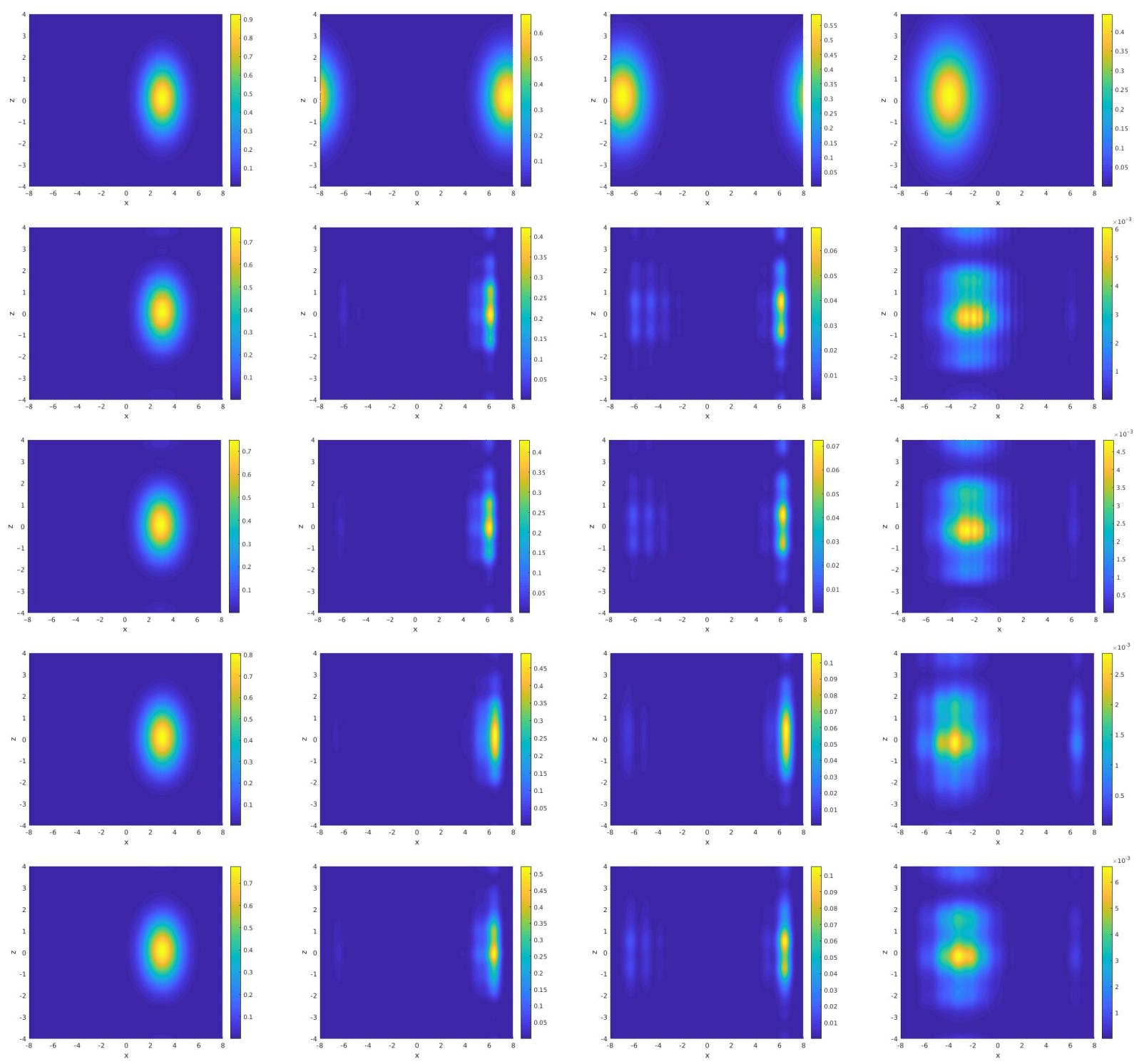

Figure 2: Test 1 : density at times $t=0.456 a$.u. (first column), $t=1.14 a . u$. (second column), $t=1.368 a . u$. (third column) $t=T=1.824 a . u$. (fourth column): with periodic boundary conditions (without PML) (first line) The second (respectively third) line reports the results for the density with TSSP-PML, with a PML of Type-III (respectively Type-IV), setting $\sigma_{0}=10^{-2}, \theta=\pi / 4$. The fourth (respectively fifth) line gives the same kind of results but for the Type-V (respectively Type-VI) PML, with $\sigma_{0}=10^{-2}, \theta=\pi / 4$ and $\delta_{\nu}=L_{\nu}-L_{\nu}^{*}$. 


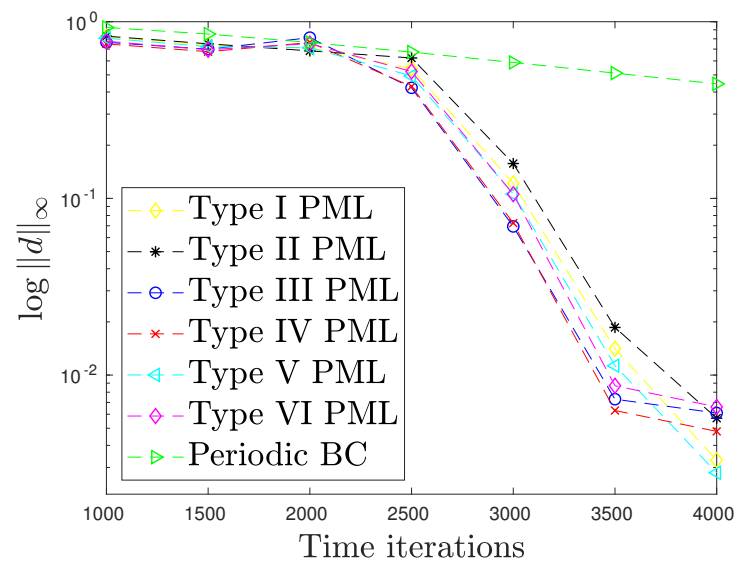

Figure 3: Test 1 : maximum of the density in logscale, as a function of the time iteration, for 6 different types of PML.
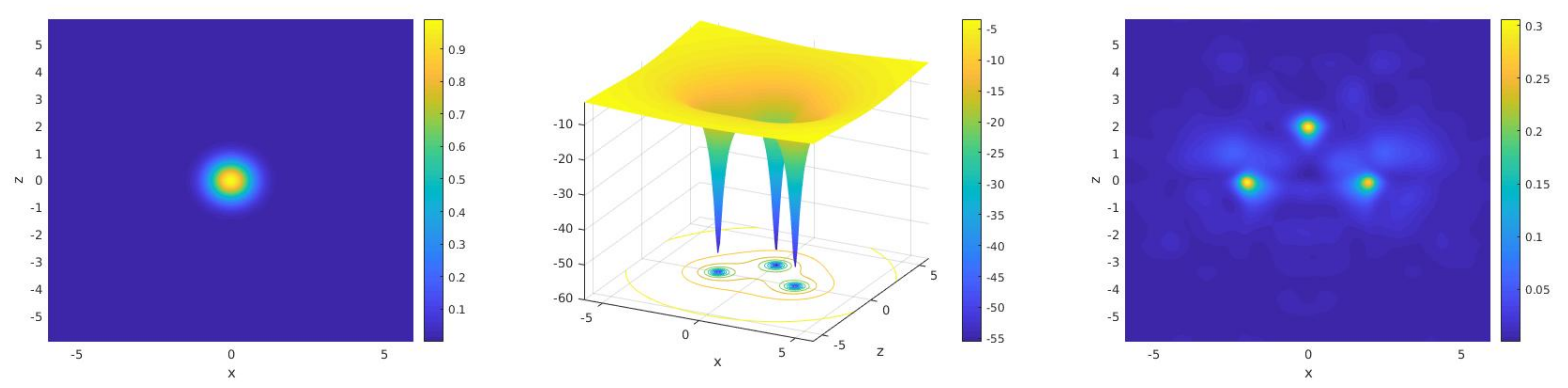

Figure 4: Test 2 : (Left) Initial density. (Middle) 3-nucleus potential. (Right) Density at time $T=$ $3 \times 10^{-1}$ a.u. without external laser field. 


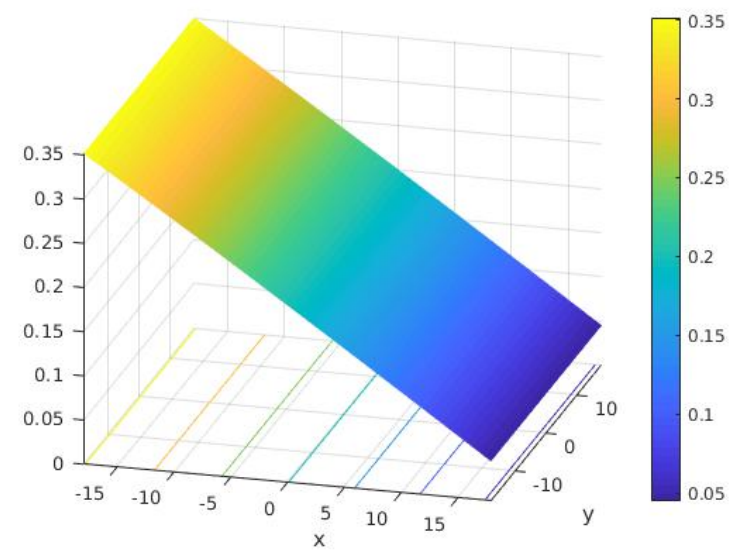

Figure 5: Test 2 : $z$-component of the external laser potential: $A_{z}(T, x, z)$ for $T=3 \times 10^{-1} a . u$.

as the Dirac wave function. Without external excitation the wavepacket is then trapped by the potential. We then plug an external laser field which drives the wavepacket: a linearly polarized electric field is imposed such that $A_{x}$ is identically null, and $A_{z}(t, x, z)$ is defined as follows

$$
A_{z}(t, x, z)=A_{0} \cos (\omega(x / c-t)) f(\omega(x / c-t))
$$

with $A_{0}=1000 / 137 a . u$., $\omega=1$, and where the envelope function is linear, then constant, and finally linear. More specifically $f$ is defined as

$$
f(t)= \begin{cases}\frac{\omega}{n \pi} t, & t \in[0, n \pi / \omega], \\ 1, & t \in\left[n \pi / \omega,\left(n+n^{\prime}\right) \pi / \omega\right], \\ \frac{\omega}{n \pi}\left(t-\frac{\left(2 n+n^{\prime}\right) \pi}{\omega}\right), & t \in\left[\left(n+n^{\prime}\right) \pi / \omega,\left(2 n+n^{\prime}\right) \pi / \omega\right],\end{cases}
$$

where $2 n+n^{\prime}$ denotes the total number of half-cycles. In the test, we took $n=n^{\prime}=1$. We report the $z$-component of the external laser potential in Fig. 5 at final time. We also include a non-null wavenumber in the initial wavepacket, $\boldsymbol{k}=(10,0)$, out of the ions influence. The wavepacket will eventually be absorbed by the PML unlike the solution without PML.

At times $t=3 \times 10^{-2}$ a.u., $t=6 \times 10^{-2}$ a.u., $t=3 \times 10^{-1}$ a.u. and $t=3.6 \times 10^{-1}$ a.u., we compare 3 solutions Fig. 6, 7, 8, 9, 1) the solution of reference computed on $[-18,18] \times$ $[-18,18], 2)$ the solution without PML, and 3) the solution with Type V-PML and $\sigma_{0}=10^{-2}$, $\theta=\pi / 4$, and $\delta_{\nu}=1.2$. At time $t=6 \times 10^{-2} a . u$., the 3 solutions look identical, as the wavefunction has not reached the boundary of the computational domain $[-6,6] \times[-6,6]$. At $t=T=3 \times 10^{-1} a$.u., we however clearly see that the wavefunction of reference has mainly left the region $[-6,6] \times[-6,6]$, while the PML solution was almost totally absorbed and the solution without PML, was maintained in the computational domain due to non-absorbing 

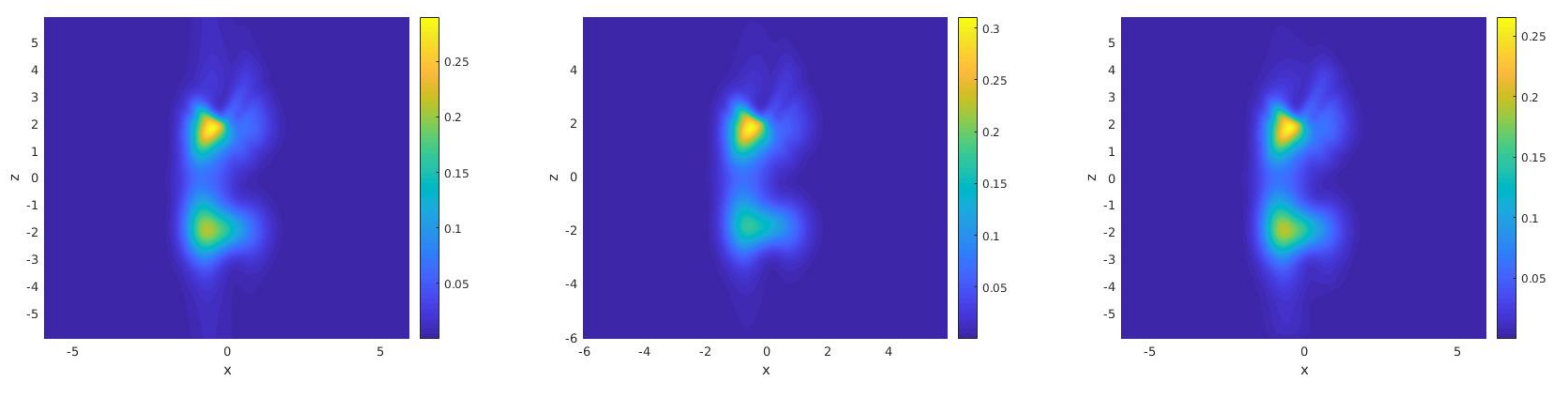

Figure 6: Test 2 : From left to right: No PML, reference and Type V-PML solutions at time $t=3 \times 10^{-2} a . u$..
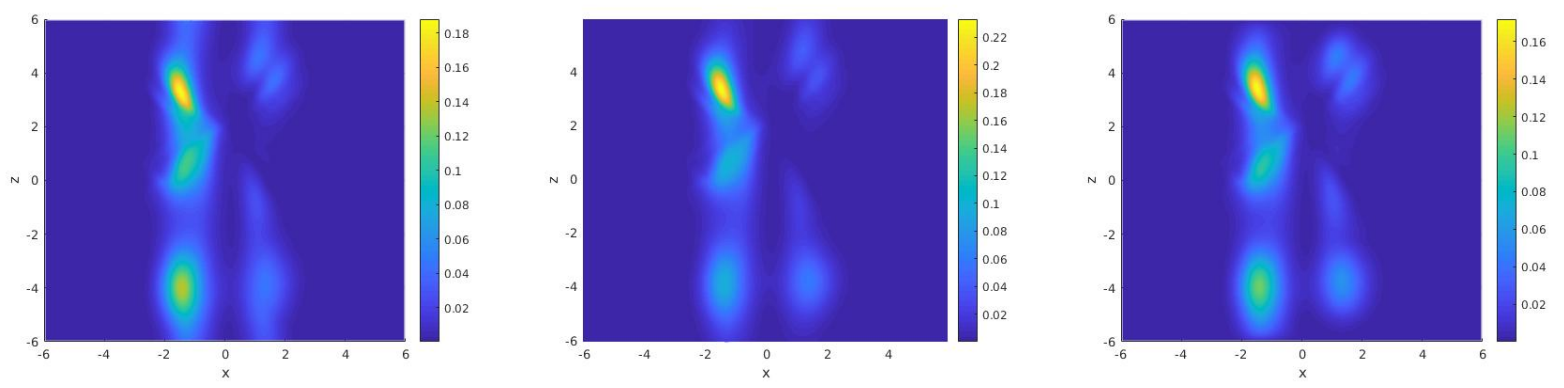

Figure 7: Test 2 : From left to right: No PML, reference and Type V-PML solutions at time $t=6 \times 10^{-2} a . u$..

boundary layers. This is also illustrated on Fig. 10, where the $\ell^{2}$-norm as function of time in the $\mathcal{D}$ is represented for the 3 solutions. We see that, unlike the solution without PML, the PML-solution makes decrease the $\ell^{2}-$ norm, thanks to the absorbing layers. We also report in Fig. 10 (Top) the solution of reference at time $T=0.3 a . u$. in a larger domain $[-18,18] \times[-18,18]$. By comparison, we see that on this example, the solution of reference has also a decreasing $\ell^{2}-$ norm in the zone $\mathcal{D}\left(\left(t,\left\|d_{\mid \mathcal{D}}^{\operatorname{Ref}}(t, \cdot)\right\|_{2}\right)\right)$, see Fig. 10 where the $\ell^{2}$ norm is represented in logscale as a function of time (Bottom). We also report the solution of reference in the domain $[-18.18] \times[-18,18]$ at time $T=0.6 a$.u.

Test 3 : evolution of wavepacket subject to a weakly nonlinear potential. We consider the two-dimensional Dirac equation with a nonlinear potential

$$
\begin{aligned}
V(x, z, \psi)= & -\frac{Z_{\mathrm{A}}}{\sqrt{\left(x-x_{\mathrm{A}}\right)^{2}+\left(z-z_{\mathrm{A}}\right)^{2}+1}} \\
& -\frac{Z_{\mathrm{B}}}{\sqrt{\left(x-x_{\mathrm{B}}\right)^{2}+\left(z-z_{\mathrm{B}}\right)^{2}+1}}+|\psi|^{2},
\end{aligned}
$$

with $Z_{\mathrm{A}}=Z_{\mathrm{B}}=2,\left(x_{\mathrm{A}}, z_{\mathrm{A}}\right)=(-1,4)$ and $\left(x_{\mathrm{B}}, z_{\mathrm{B}}\right)=(1,4)$. The initial density with wave vector $\boldsymbol{k}=(2,10)$ is represented in Fig. 11. More specifically, the initial data is given by

$$
\boldsymbol{\psi}(x, y, z, 0)=\mathcal{N}[1,0,0,0]^{T} \exp \left(-\left(x^{2}+y^{2}+z^{2}\right) / \Delta^{2}\right) \times \exp \left(\mathrm{i} k_{x} x\right),
$$



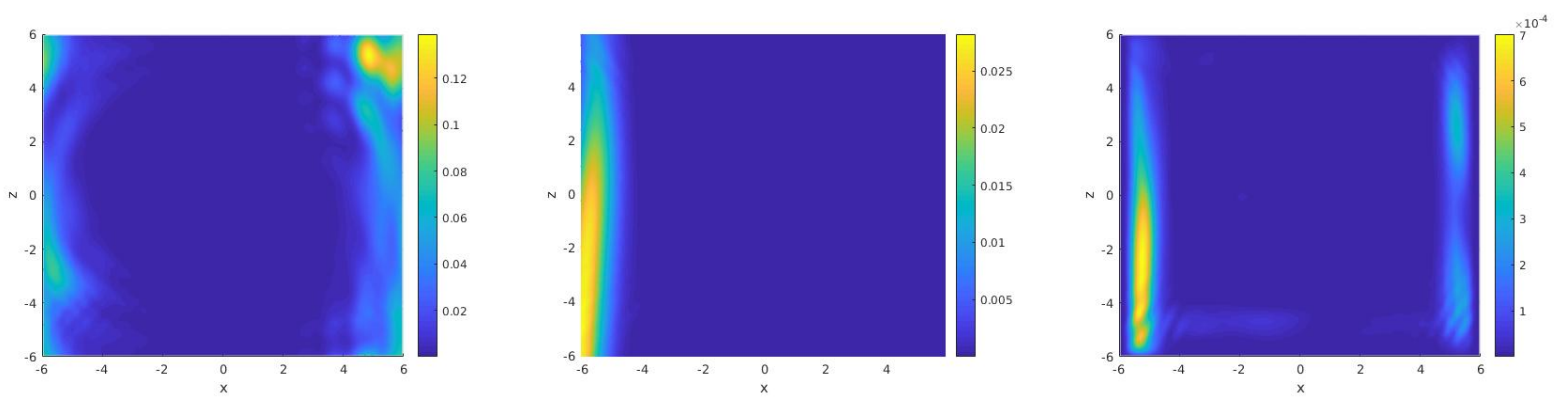

Figure 8: Test 2 : From left to right: No PML, reference and Type V-PML solutions at time $t=3 \times 10^{-1} a . u$..
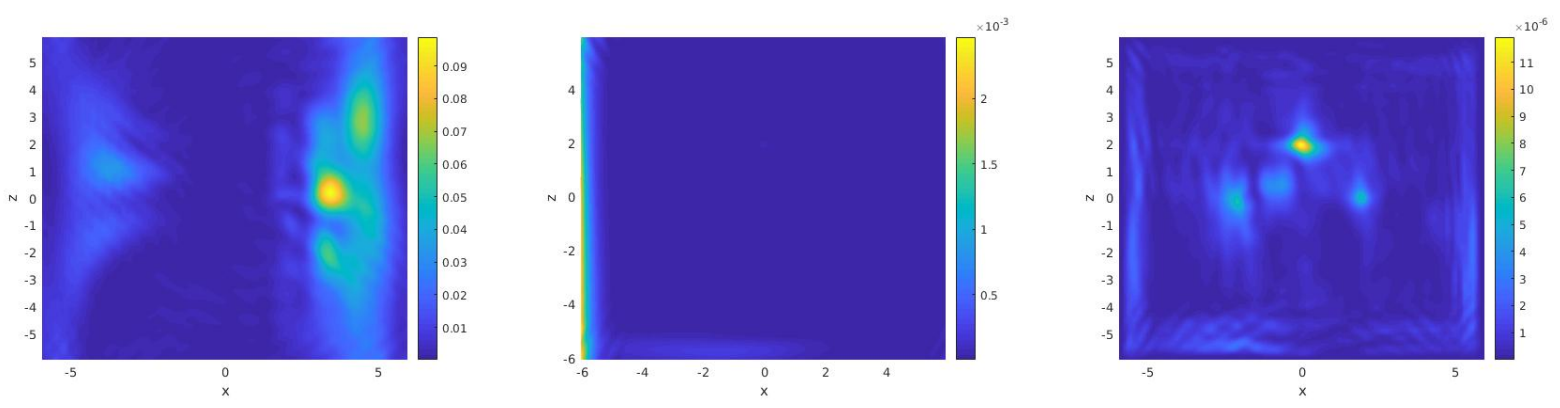

Figure 9: Test 2 : From left to right: No PML, reference and Type V-PML solutions at time $t=3.6 \times$ $10^{-1}$ a.u..
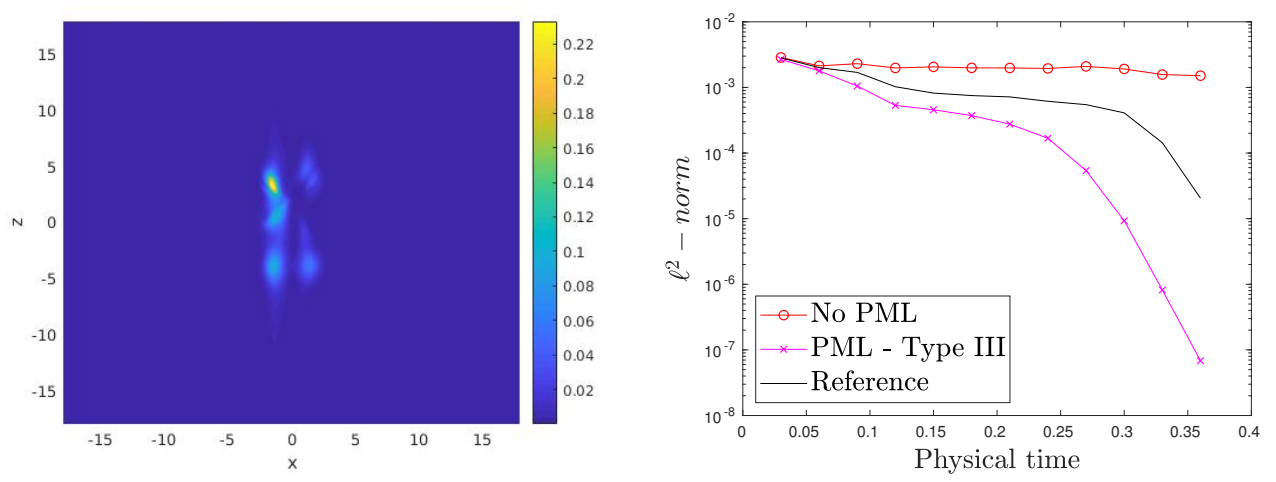

Figure 10: Test 2 : (Top) Solution of reference at time $T=6 \times 10^{-2} a . u$. in the domain $[-18,18] \times[-18,18]$. (Bottom) $\ell^{2}$-norm in logscale of the 3 solutions (No PML, Reference, Type V-PML) as a function of time in $\mathcal{D}$. 
where $\Delta=128$ in $(37)$ and $\mathcal{N}$ is a normalization coefficient, such that $\|\psi(\cdot, 0)\|_{\left(L^{2}(\mathcal{D})\right)^{4}}=1$. The overall computational domain is $\mathcal{D}=[-8,8]^{2}$, with $N_{x} \times N_{y}=256^{2}$ grid points and $\Delta t=4.56 \times 10^{-4}$ a.u. . We report on Fig. 12 the total density at time $T=0.912 a . u$. in logscale, i.e. $\log \left(d(T, x, z)\right.$, for $(x, z) \in \mathcal{D}$, where $d(T, x, z)=\sum_{i=1}^{4}\left|\psi_{i}(T, x, z)\right|^{2}$, setting $\boldsymbol{\psi}=\left(\psi_{1}, \psi_{2}, \psi_{3}, \psi_{4}\right)$ as the Dirac wave function. At that time, the wavepacket passed through the boundary of the computational domain at $z= \pm 8 a$.u. The nonlinearity is numerically treated explicitly. The use of the logscale allows to fairly report the accuracy of the PML. In (32), we take $L_{\nu}=8 a . u$., $L_{\nu}^{*}=0.8 L_{\nu}$ a.u., $\theta=\pi / 4$ (similarly to the Schrödinger equation [5, 109]), and we pick the Type-IV PML, i.e. $\sigma: \nu \mapsto \sigma_{0} / \nu^{2}$ (singular profile [5]). We represent the solution with different values of $\sigma_{0}$. These results show that the PML properly absorbs except for $\sigma_{0}=5$, with reflection magnitude as weak as $10^{-6}$ for $\sigma_{0}=10^{-2}$. Let us remark that other PMLs could be considered as well [88], but recall that we "only" intend here to prove the feasibility of the method for various PMLs. For completeness and by comparisons with results obtained in Fig. 12, we also report in Fig. 13 the solution to the Dirac equation in $\mathcal{D}$ with zero Dirichlet boundary condition by using the real-space quantum lattice Boltztmann method proposed in [43] and illustrating the total wave reflection at the domain boundary when PMLs or ABCs are not used. This simple example illustrates the fact without using PML and or low-order ABC, a wavefunction is totally reflected. This is a standard issue when solving the Dirac equation, in particular when studying laser-molecule interaction. We report in Fig. 16 (Top), the maximum of the density as a function of time for $\sigma_{0}=10^{-3}, 10^{-2}, 10^{-1}, 1,5$ in logscale with the PML of Type IV for $\theta=\pi / 4$. We clearly see that $\sigma_{0}=10^{-2}$ provides the best absorption properties. We next compare in Fig. 14, for $\sigma_{0}=10^{-2}$, the reflection for $\theta=\pi / 16, \theta=\pi / 8, \theta=\pi / 4$ and $\theta=\pi / 2$ showing the importance of properly selecting $\theta$; the best absorption is obtained with $\theta=\pi / 16$. We report in Fig. 16 (Bottom), the maximum of the density as a function of the time iteration for different values of $\theta=\pi / 16, \pi / 8, \pi / 4, \pi / 2$ with Type IV-PML. We again see an optimal performance with $\theta=\pi / 16$ but $\theta=\pi / 8$ or $\theta=\pi / 4$ also provides some good results, meaning that the PML is relatively stable with $\theta$. In the last test, we compare in Fig. 15, the efficiency of the PML for four different absorbing functions (Types I to IV), with respectively $\sigma(\nu)=\sigma_{0}\left(\nu+\delta_{\nu}\right)^{2}$, $\sigma(\nu)=\sigma_{0}\left(\nu+\delta_{\nu}\right)^{3}, \sigma(\nu)=-\sigma_{0} / \nu$ and $\sigma(\nu)=\sigma_{0} / \nu^{2}$. We see that the best results are obtained for the Type-IV PML, a similar quality being also obtained for the Types $\mathrm{V}$ and VI PMLs (not reported here).

These tests clearly show the relevance of the combination of the TSSP method with PMLs, as we simultaneously benefit from the efficiency and accuracy of FFTs and the high absorption feature of PMLs to avoid reflections at the boundary.

Test 4 : convergence. In this last test, we propose a simple convergence benchmark. A wavepacket with wavenumber $\boldsymbol{k}=(5,0)$ is propagating in vacuum. We plot the initial density, and final solution with periodic conditions on $\mathcal{D}=[-8,8]^{2}$ in Fig. 17 (Left, Middle). We report the supremum of the solution for $N_{x}=N_{y}=32,256,320,512,640$, 768 with the PML of Type-III, for $\sigma_{0}=10^{-2}, \theta=\pi / 4$, fixing the time step to $\Delta t=1.8 \times 10^{-3} a . u$.. We report in Fig. 17 (Right), the maximum of the density at final time $T$ in $\operatorname{logscale}$ $\log \left(\sup _{(x, z) \in[-8,8]^{2}}|d(T, x, z)|\right)$ as a function of the number of gridpoints. We observe an 

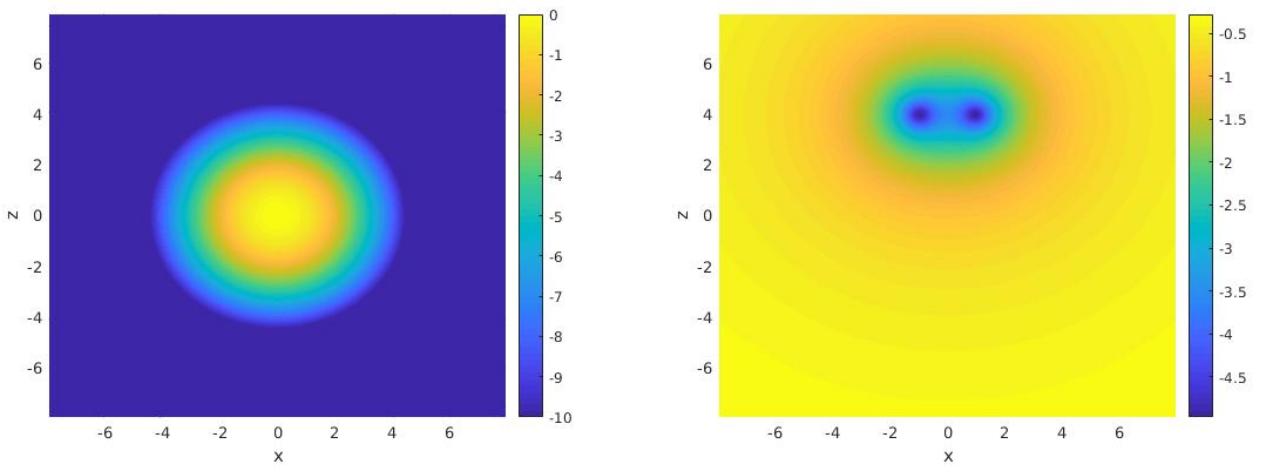

Figure 11: Test 3 : initial density in logscale (Top) and static potential (Bottom).
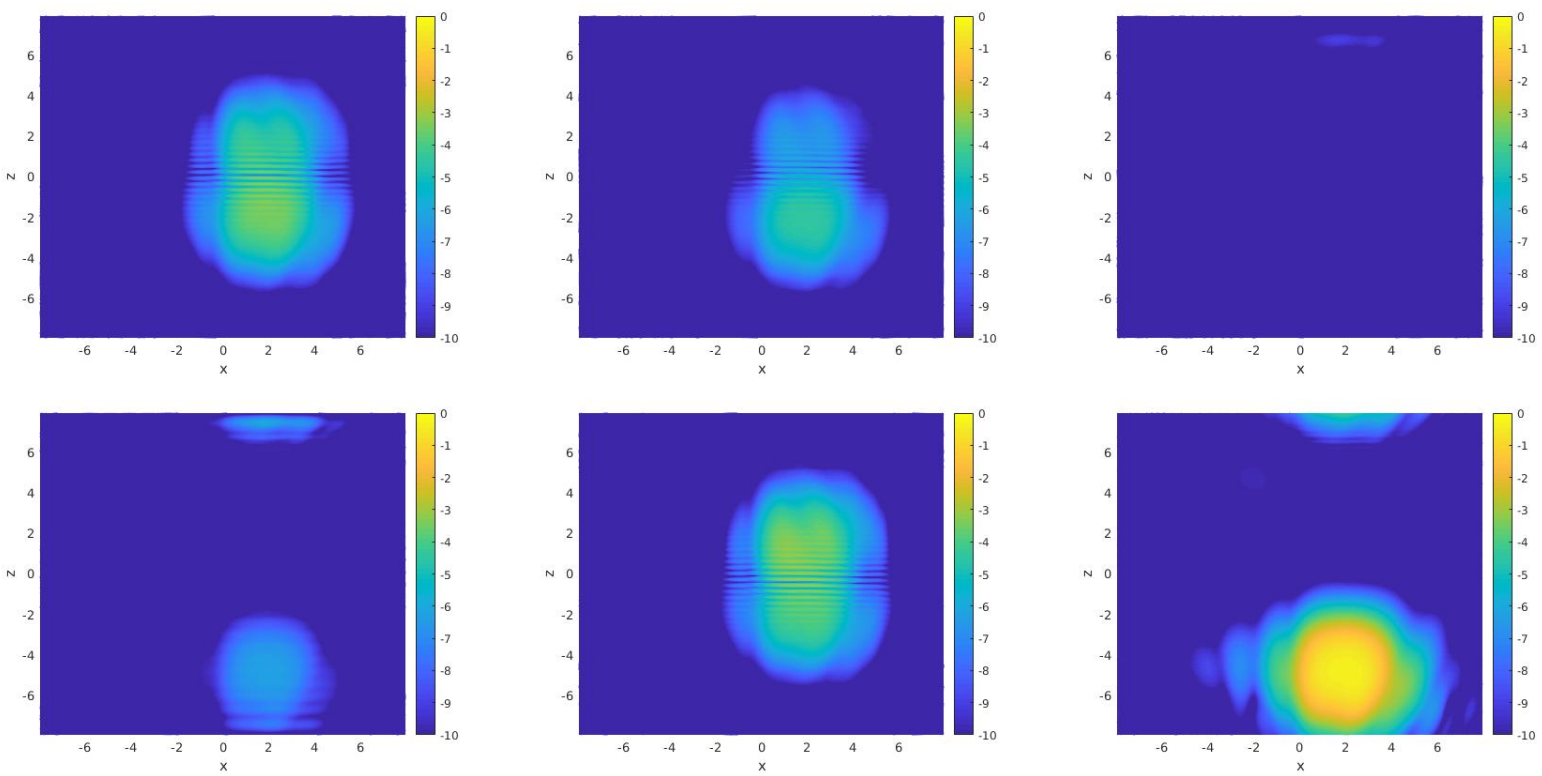

Figure 12: Test 3 : logarithm of the density at time $T=0.912$ (from top-left to bottom-right) for the PML with parameters $\sigma_{0}=1, \sigma_{0}=10^{-1}, \sigma_{0}=10^{-2}, \sigma_{0}=10^{-3}, \sigma_{0}=5$, and finally with periodic boundary conditions $(S=1)$. 


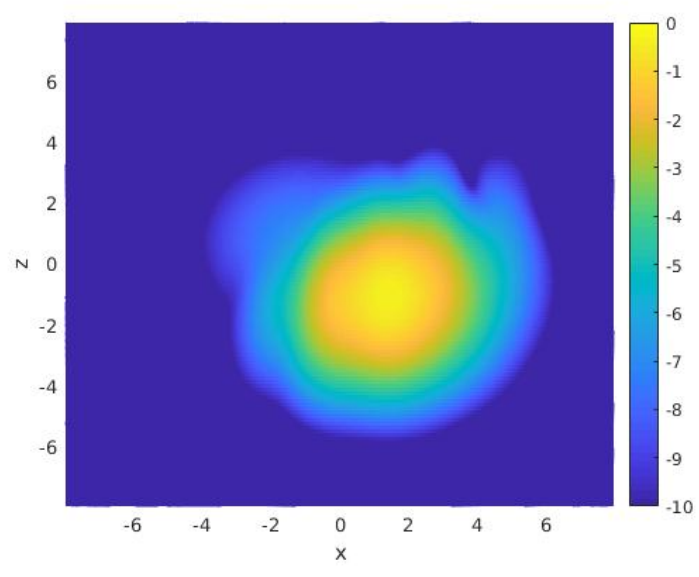

Figure 13: Test 3 : logarithm of the density at time $T=0.912 a . u$. with null Dirichlet boundary condition. This test illustrates that artificial wave-reflecting occurs with real space methods (here a quantum lattice Boltzmann method [43]) are implemented without PML or ABC.

accurate convergence up to a certain precision. Notice that what is reported is the maximum of the solution over the physical domain, including the PML. In other words the smaller the maximum, the better the absorption. The saturation error comes from the limit of the PML accuracy and error in time (second-order splitting). This shows the high accuracy (spectral-like) of the TSSP with PML.

\section{Conclusion}

In this paper, we have proposed a simple time-splitting pseudospectral method which allows for the numerical computation of IBVP for the Dirac equation with efficient PML. Some numerical experiments have shown that the method preserves the accuracy of the PML with static or time-dependent external potentials. Different absorbing functions were considered and tested. The implementation strategy is developed for the TSSP approximation, but can be directly extended to other numerical schemes including implicit ones. We think that Fourier-based codes solving the time-dependent Dirac equation can easily be adapted to include the absorbing layers, hence drastically reducing the negative effect of periodic conditions and simultaneously avoiding artificial wave reflections. We next plan to analyze mathematically the stability and convergence of the method as well as the PML accuracy for different types of absorbing functions.

Acknowledgments. X. ANTOINE was partially supported by the French National Research Agency project NABUCO, grant ANR-17-CE40-0025. E. LORIN received support from NSERC through the Discovery Grant program.

\section{References.}



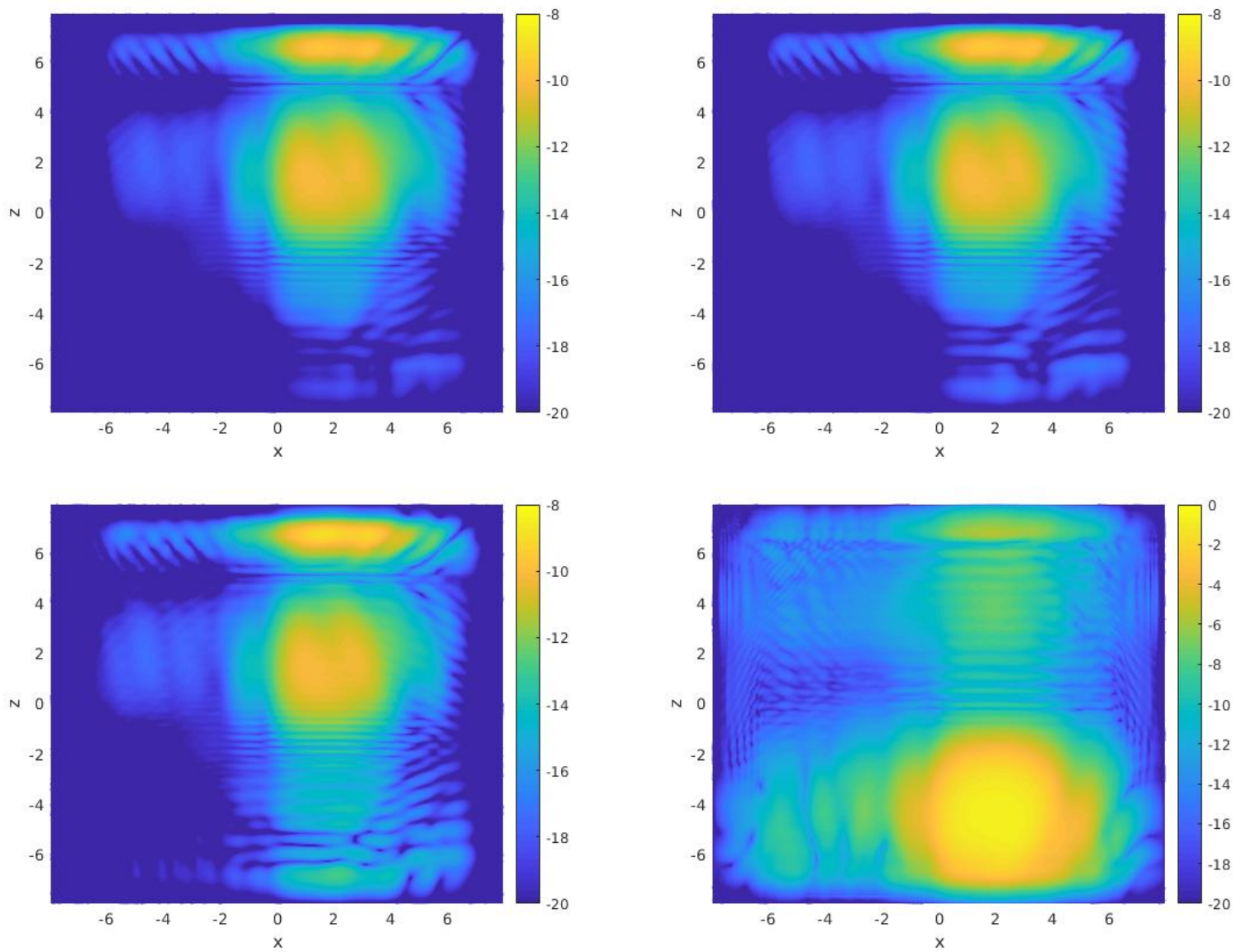

Figure 14: Test 3 : logarithm of the density at time $T=0.912 a . u$. with $\sigma_{0}=10^{-2}$ and from top-left to right-bottom: $\theta=\pi / 16, \theta=\pi / 8, \theta=\pi / 4$ and $\theta=\pi / 2$. 

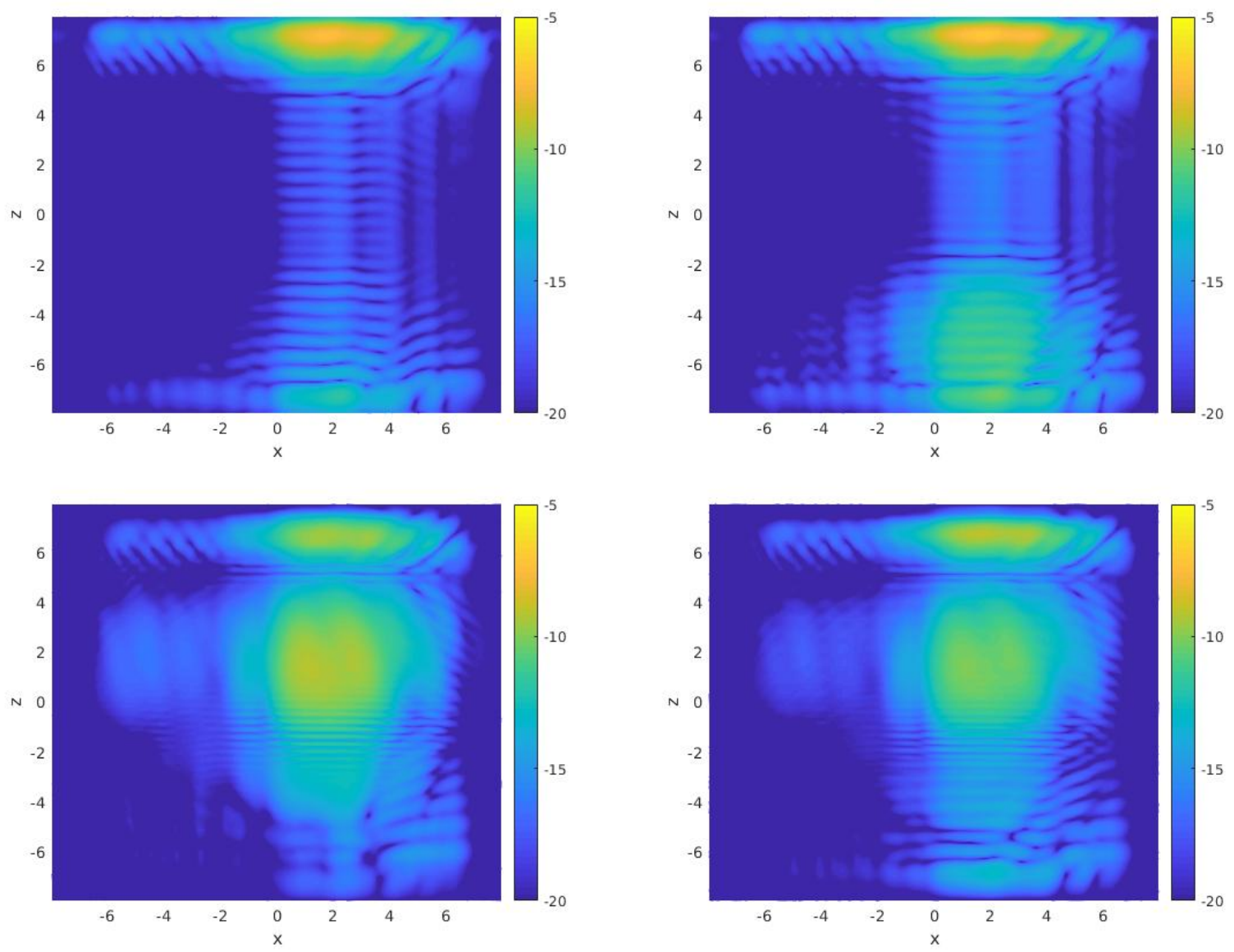

Figure 15: Test 3 : logarithm of the density at time $T=0.912 a . u$. for $\sigma_{0}=10^{-2} \theta=\pi / 4, \delta_{\nu}=L_{\nu}-L_{\nu}^{*}$, and from top-left to right-bottom: absorption functions $\sigma$ of type I to IV.
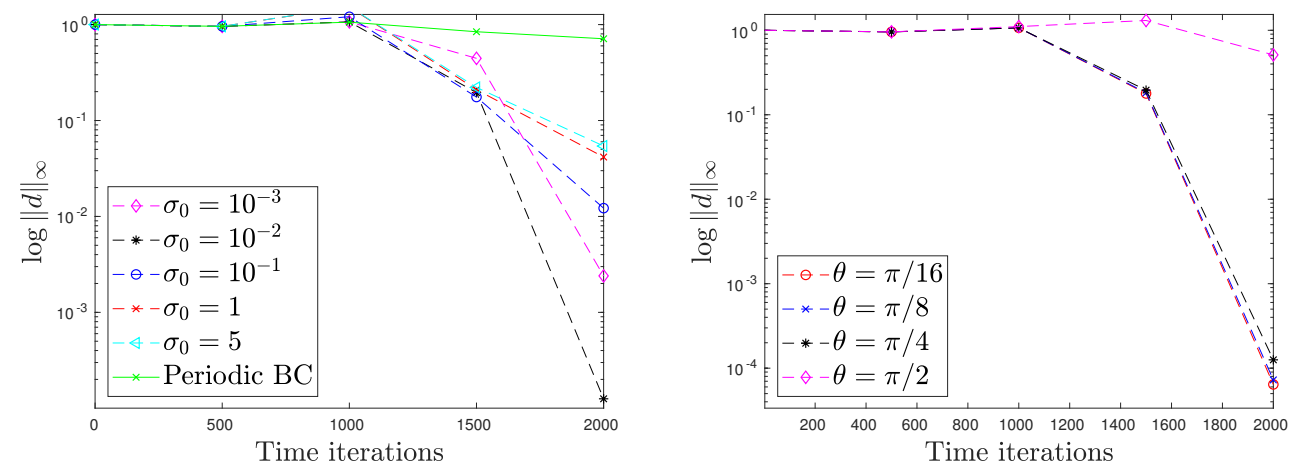

Figure 16: Test 3 : PML of type IV (Top) maximum of the density as a function of the time iteration with 5 different values of $\sigma$ and for $\theta=\pi / 4$; (Bottom) maximum of the density as a function of the time iteration for $\theta=\pi / 16, \pi / 8, \pi / 4, \pi / 2$ and $\sigma_{0}=10^{-2}$. 

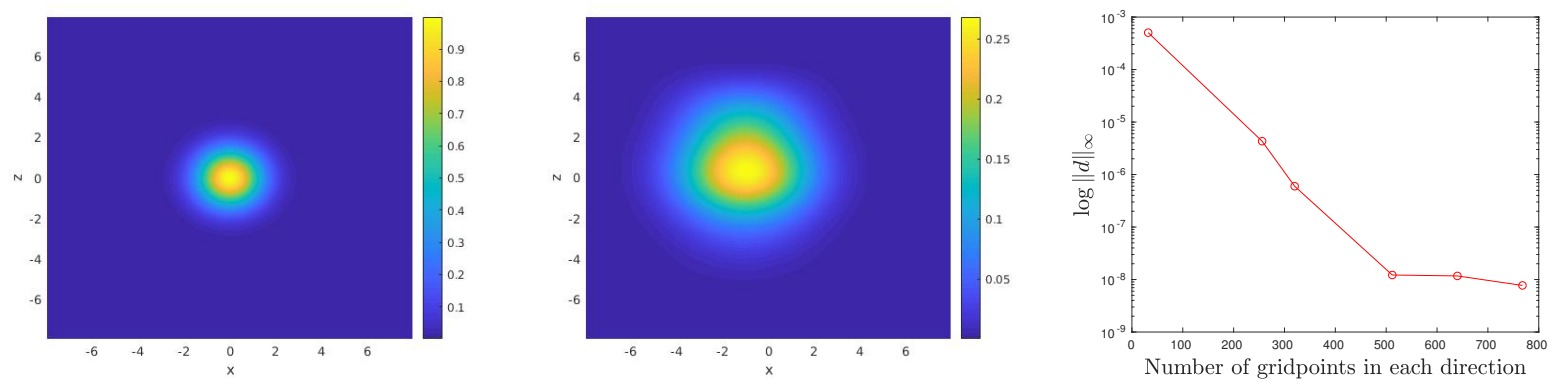

Figure 17: Test 3 : initial data (Left), periodic boundary conditions (Middle), convergence with the Type-III PML (Right), for $\sigma_{0}=10^{-2}, \theta=\pi / 4$ and $N_{x}=N_{y}=32,256,320,512,640,768$.

[1] E. Ackad and M. Horbatsch. Numerical solution of the Dirac equation by a mapped Fourier grid method. J. of Phys. A: Mathematical and General, 38(14):3157, 2005.

[2] E. Ackad and M. Horbatsch. Calculation of electron-positron production in supercritical uranium-uranium collisions near the Coulomb barrier. Phys. Rev. A, 78:062711, Dec 2008.

[3] X. Antoine, A. Arnold, C. Besse, M. Ehrhardt, and A. Schädle. A review of transparent and artificial boundary conditions techniques for linear and nonlinear Schrödinger equation. Commun. Comput. Phys., 4(4):729-796, 2008.

[4] X. Antoine, C. Besse, and V. Rispoli. High-order IMEX-spectral schemes for computing the dynamics of systems of nonlinear Schrödinger/Gross - Pitaevskii equations. J. Comput. Phys., 327:252-269, 2016.

[5] X. Antoine, C. Geuzaine, and Q. Tang. Perfectly Matched Layer for computing the dynamics of nonlinear Schrödinger equations by pseudospectral methods. Application to rotating Bose-Einstein condensates. In preparation, 2018.

[6] X. Antoine and E. Lorin. Computational performance of simple and efficient sequential and parallel Dirac equation solvers. Comput. Phys. Commun., 220:150-172, 2017.

[7] X. Antoine, E. Lorin, J. Sater, F. Fillion-Gourdeau, and A.D. Bandrauk. Absorbing boundary conditions for relativistic quantum mechanics equations. J. Comput. Phys., 277:268-304, 2014.

[8] X. Antoine, E. Lorin, and Q. Tang. A friendly review of absorbing boundary conditions and perfectly matched layers for classical and relativistic quantum waves equations. Molecular Physics, 115(15-16):1861-1879, 2017.

[9] A. N. Artemyev, A. Surzhykov, P. Indelicato, G. Plunien, and Th. Stöhlker. Finite basis set approach to the two-centre Dirac problem in Cassini coordinates. J. of Phys. B: Atomic, Molecular and Optical Physics, 43(23):235207, 2010. 
[10] S. Balay, W. D. Gropp, L. Curfman McInnes, and B. F. Smith. Efficient management of parallelism in object oriented numerical software libraries. In E. Arge, A. M. Bruaset, and H. P. Langtangen, editors, Modern Software Tools in Scientific Computing, pages 163-202. Birkhäuser Press, 1997.

[11] A.D. Bandrauk and H. Shen. Improved exponential split operator method for solving the time-dependent Schrödinger equation. Chem. Phys. Lett., 176(5):428 - 432, 1991.

[12] W. Bao, Y. Cai, X. Jia, and Q. Tang. Numerical methods and comparison for the Dirac equation in the nonrelativistic limit regime. J. Sc. Comput., 71(3):1-41, 2017.

[13] W. Bao and X.-G. Li. An efficient and stable numerical method for the Maxwell-Dirac system. J. Comput. Phys., 199(2):663-687, 2004.

[14] W. Bao and X.-G. Li. An efficient and stable numerical method for the Maxwell-Dirac system. J. Comput. Phys., 199(2):663-687, 2004.

[15] C. Bardos and E. Tadmor. Stability and spectral convergence of Fourier method for nonlinear problems: on the shortcomings of the $2 / 3$ de-aliasing method. Numer. Math., 129(4):749-782, 2015.

[16] H. Bauke and C.H. Keitel. Accelerating the Fourier split operator method via graphics processing units. Comput. Phys. Commun., 182(12):2454-2463, 2011.

[17] P.E. Bechouche, N. J. Mauser, and S. Selberg. On the asymptotic analysis of the DiracMaxwell system in the nonrelativistic limit. J. Hyperbolic Differ. Equ., 2(1):129-182, 2005.

[18] U. Becker, N. Grun, and W. Scheid. Solution of the time-dependent Dirac equation by the finite difference method and application for $\mathrm{Ca}^{20+}+\mathrm{U}^{91+}$. J. of Phys. B: Atomic and Molecular Physics, 16(11):1967, 1983.

[19] R. Beerwerth and H. Bauke. Krylov subspace methods for the Dirac equation. Comput. Phys. Commun., 188:189 - 197, 2015.

[20] A. Bermúdez, L. Hervella-Nieto, A. Prieto, and R. Rodríguez. An exact bounded perfectly matched layer for time-harmonic scattering problems. SIAM J. Sci. Comput., 30:312-338, 2007.

[21] A. Bermúdez, L. Hervella-Nieto, A. Prieto, and R. Rodríguez. An optimal perfectly matched layer with unbounded absorbing function for time-harmonic acoustic scattering problems. J. Comput. Phys., 223:469-488, 2007.

[22] N. Bournaveas. Local existence for the Maxwell-Dirac equations in three space dimensions. Commun. Partial Differential Equations, 21(5-6):693-720, 1996. 
[23] T. Brabec and F. Krausz. Intense few-cycle laser fields: Frontiers of nonlinear optics. Rev. Mod. Phys., 72(2):545-591, Apr 2000.

[24] J. W. Braun, Q. Su, and R. Grobe. Numerical approach to solve the time-dependent Dirac equation. Phys. Rev. A, 59(1):604-612, Jan 1999.

[25] J. M. Chadam. Global solutions of the Cauchy problem for the (classical) coupled Maxwell-Dirac equations in one space dimension. J. Funct. Anal., 13:173-184, 1973.

[26] J. Chazarain and A. Piriou. Introduction to the theory of linear partial differential equations, volume 14 of Studies in Mathematics and its Applications. North-Holland Publishing Co., Amsterdam, 1982. Translated from the French.

[27] C. C. Chen, H. P. Chang, and C. S. Hsue. A highly accurate calculation for the electronic states of $\mathrm{H}+2$ in B-spline basis. Chem. Phys. Lett., 217(5-6):486 - 489, 1994.

[28] W.C. Chew and Q.H. Liu. Perfectly matched layers for elastodynamics: A new absorbing boundary condition. J. Comput. Acoustics, 4(4):341-359, 1996.

[29] F. Collino and P. Monk. The perfectly matched layer in curvilinear coordinates. SIAM J. Sci. Comput., 19:2061-2090, 1998.

[30] T. Colonius. Modeling artificial boundary conditions for compressible flow. Annual Review of Fluid Mechanics, 36:315-345, 2004.

[31] C. Debiez, A. Dervieux, K. Mer, and B. Nkonga. Computation of unsteady flows with mixed finite volume/finite element upwind methods. Internat. J. Numer. Methods Fluids, 27(1-4, Special Issue):193-206, 1998. Finite elements in fluids.

[32] J. P. Desclaux, J. Dolbeault, M. J. Esteban, P. Indelicato, and E. Séré. Computational approaches of relativistic models in quantum chemistry. In Handbook of numerical analysis, Vol. X, Handb. Numer. Anal., X, pages 453-483. North-Holland, Amsterdam, 2003.

[33] A. Di Piazza, C. Müller, K. Z. Hatsagortsyan, and C. H. Keitel. Extremely highintensity laser interactions with fundamental quantum systems. Rev. Mod. Phys., 84:1177-1228, Aug 2012.

[34] J. Dolbeault, M. J. Esteban, and E. Séré. On the eigenvalues of operators with gaps. application to Dirac operators. J. of Funct. Anal., 174(1):208 - 226, 2000.

[35] G. W. F. Drake and S. P. Goldman. Application of discrete-basis-set methods to the Dirac equation. Phys. Rev. A, 23:2093-2098, May 1981.

[36] G. V. Dunne, H. Gies, and R. Schützhold. Catalysis of Schwinger vacuum pair production. Phys. Rev. D, 80(11):111301, Dec 2009. 
[37] A. Ern and J.-L. Guermond. Éléments finis: théorie, applications, mise en œuvre, volume 36 of Mathématiques and Applications (Berlin) [Mathematics and Applications]. Springer-Verlag, Berlin, 2002.

[38] F. H. M. Faisal. A four-component Dirac theory of ionization of a Hydrogen molecular ion in a super-intense laser field. J. Phys. B: At. Mol. Opt. Phys., 42(171003), 2009.

[39] C. Farrell and U. Leonhardt. The perfectly matched layer in numerical simulations of nonlinear and matter waves. J. of Opt. B-Quantum and Semiclassical Optics, 7(1):1-4, 2005 .

[40] F. Fillion-Gourdeau, P. Blain, D. Gagnon, C. Lefebvre, and S. Maclean. Numerical computation of dynamical Schwinger-like pair production in graphene. Russian Physics Journal, 59(11):1875-1880, 2017.

[41] F. Fillion-Gourdeau, D. Gagnon, C. Lefebvre, and S. Maclean. Time-domain quantum interference in graphene. Phys. Rev. B, 94(12), 2016.

[42] F. Fillion-Gourdeau, H. J. Herrmann, M. Mendoza, S. Palpacelli, and S. Succi. Formal analogy between the Dirac equation in its Majorana form and the discrete-velocity version of the Boltzmann kinetic equation. Phys. Rev. Lett., 111:160602, Oct 2013.

[43] F. Fillion-Gourdeau, E. Lorin, and A. D. Bandrauk. Numerical solution of the timedependent Dirac equation in coordinate space without fermion-doubling. Comput. Phys. Commun., 183(7):1403 - 1415, 2012.

[44] F. Fillion-Gourdeau, E. Lorin, and A. D. Bandrauk. A split-step numerical method for the time-dependent Dirac equation in 3-D axisymmetric geometry. J. Comput. Phys., 272:559-587, 2014.

[45] F. Fillion-Gourdeau, E. Lorin, and A.D. Bandrauk. Numerical solution of the timeindependent Dirac equation for diatomic molecules: B-splines without spurious states. Phys. Rev. A - Atomic, Molecular, and Optical Physics, 85(2), 2012.

[46] F. Fillion-Gourdeau, E. Lorin, and A.D. Bandrauk. Enhanced Schwinger pair production in many-centre systems. J. of Phys. B: Atomic, Molecular and Optical Physics, 46(17), 2013.

[47] F. Fillion-Gourdeau, E. Lorin, and A.D. Bandrauk. Resonantly enhanced pair production in a simple diatomic model. Phys. Rev. Lett., 110(1), 2013.

[48] F. Fillion-Gourdeau, E. Lorin, and A.D. Bandrauk. A split-step numerical method for the time-dependent Dirac equation in 3-d axisymmetric geometry. J. Comput. Phys., 272:559-587, 2014. 
[49] F. Fillion-Gourdeau, E. Lorin, and A.D. Bandrauk. Galerkin method for unsplit 3-d Dirac equation using atomically/kinetically balanced B-spline basis. J. Comput. Phys., 307:122-145, 2016.

[50] C. Fischer and O. Zatsarinny. A B-spline Galerkin method for the Dirac equation. Comput. Phys. Commun., 180(6):879-886, 2009.

[51] M. Flato, J. C. Simon, and E. Taflin. Asymptotic completeness, global existence and the infrared problem for the Maxwell-Dirac equations. Mem. Amer. Math. Soc., 127(606):x+311, 1997.

[52] F. Gelis, K. Kajantie, and T. Lappi. Quark-antiquark production from classical fields in heavy-ion collisions: $1+1$ dimensions. Phys. Rev. C, 71(2):024904, Feb 2005.

[53] E. Godlewski and P.-A. Raviart. Numerical approximation of hyperbolic systems of conservation laws, volume 118 of Applied Mathematical Sciences. Springer-Verlag, New York, 1996.

[54] A. Gonoskov, I. Gonoskov, C. Harvey, A. Ilderton, A. Kim, M. Marklund, G. Mourou, and A. Sergeev. Probing nonperturbative QED with optimally focused laser pulses. Phys. Rev. Lett., 111:060404, Aug 2013.

[55] J. Goodman, T. Hou, and E. Tadmor. On the stability of the unsmoothed Fourier method for hyperbolic equations. Numer. Math., 67(1):93-129, 1994.

[56] I. P. Grant. Conditions for convergence of variational solutions of Dirac's equation in a finite basis. Phys. Rev. A, 25:1230-1232, Feb 1982.

[57] I. P. Grant. Variational methods for Dirac wave equations. J. of Phys. B: Atomic and Molecular Physics, 19(20):3187, 1986.

[58] I. P. Grant. Relativistic Quantum Theory of Atoms and Molecules: Theory and Computation. Springer Series on Atomic, Optical, and Plasma Physics. Springer, 2006.

[59] I. P. Grant. B-spline methods for radial Dirac equations. J. of Phys. B: Atomic, Molecular and Optical Physics, 42(5):055002, 2009.

[60] I. P. Grant and H. M. Quiney. Rayleigh-Ritz approximation of the Dirac operator in atomic and molecular physics. Phys. Rev. A, 62:022508, Jul 2000.

[61] W. Greiner. Relativistic Quantum Mechanics, Wave Equations. Springer, 1987.

[62] L. Gross. The Cauchy problem for the coupled Maxwell and Dirac equations. Commun. Pure Appl. Math., 19:1-15, 1966.

[63] B.-Y. Guo, J. Shen, and C.-L. Xu. Spectral and pseudospectral approximations using Hermite functions: Application to the Dirac equation. Adv. in Comput. Math., 19(1$3): 35-55,2003$. 
[64] R. Hammer, W. Pötz, and A. Arnold. A dispersion and norm preserving finite difference scheme with transparent boundary conditions for the Dirac equation in $(1+1) \mathrm{d} . \quad J$. Comput. Phys., 256:728-747, 2014.

[65] V. Hernandez, J. E. Roman, and V. Vidal. SLEPc: A scalable and flexible toolkit for the solution of eigenvalue problems. ACM Trans. Math. Software, 31(3):351-362, 2005.

[66] F.Q. Hu. On absorbing boundary conditions for linearized Euler equations by a perfectly matched layer. J. Comput. Phys., 129(1):201-219, 1996.

[67] F.Q. Hu. A stable, perfectly matched layer for linearized Euler equations in unsplit physical variables. J. Comput. Phys., 173(2):455-480, 2001.

[68] Z. Huang, S. Jin, P. A. Markowich, C. Sparber, and C. Zheng. A time-splitting spectral scheme for the Maxwell-Dirac system. J. Comput. Phys., 208(2):761-789, 2005.

[69] C. Itzykson and J. B. Zuber. Quantum Field Theory. McGraw-Hill, 1980.

[70] M. I. Katsnelson, K. S. Novoselov, and A. K. Geim. Chiral tunnelling and the Klein paradox in graphene. Nature Physics, 2:620 - 625, 2006.

[71] D. Kincaid and W. Cheney. Numerical analysis. Brooks/Cole Publishing Co., Pacific Grove, CA, second edition, 1996. Mathematics of scientific computing.

[72] T. Kjellsson, M. Førre, A.S. Simonsen, S. Selstø, and E. Lindroth. Alternative gauge for the description of the light-matter interaction in a relativistic framework. Phys. Rev. A, 96(2), 2017.

[73] T. Kjellsson, S. Selst $\varnothing$, S. Brck, and E. Lindroth. Relativistic dynamics for hydrogenlike systems. volume 635, 2015.

[74] O. Kullie, C. Dusterhoft, and D. Kolb. Dirac-Fock finite element method (FEM) calculations for some diatomic molecules. Chemical Physics Letters, 314(3-4):307 310, 1999.

[75] W. Kutzelnigg. Basis set expansion of the dirac operator without variational collapse. International J. of Quantum Chemistry, 25(1):107-129, 1984.

[76] Leif Laaksonen and Ian P. Grant. Two-dimensional fully numerical solutions of molecular Dirac equations. one-electron molecules. Chem. Phys. Lett., 109(5):485 - 487, 1984.

[77] J.-L. Lions and E. Magenes. Non-homogeneous boundary value problems and applications. Vol. II. Springer-Verlag, New York, 1972. Translated from the French by P. Kenneth, Die Grundlehren der mathematischen Wissenschaften, Band 182. 
[78] E. Lorin and A. Bandrauk. A simple and accurate mixed $P_{0}-Q_{1}$ solver for the MaxwellDirac equations. Nonlin. Anal. Real World Appl., 12(1):190-202, 2011.

[79] E. Lorin and A. D. Bandrauk. Multiresolution scheme for time dependent Schrödinger equations. Comput. Phys. Commun., 181(3):626-638, 2010.

[80] E. Lorin, S. Chelkowski, and A. Bandrauk. A numerical Maxwell-Schrödinger model for laser-matter interaction and propagation. Comput. Phys. Commun., 177(12):908-932, 2007.

[81] N. J. Mauser and S. Selberg. Convergence of the Dirac-Maxwell system to the VlasovPoisson system. Commun. Partial Differential Equations, 32(1-3):503-524, 2007.

[82] N. Milosevic, V. P. Krainov, and T. Brabec. Semiclassical Dirac theory of tunnel ionization. Phys. Rev. Lett., 89(19):193001, Oct 2002.

[83] G. R. Mocken and C. H. Keitel. Quantum dynamics of relativistic electrons. J. Comput. Phys., 199(2):558 - 588, 2004.

[84] G. R. Mocken and C. H. Keitel. FFT-split-operator code for solving the Dirac equation in 2+1 dimensions. Comput. Phys. Commun., 178(11):868-882, 2008.

[85] B. Müller, J. Rafelski, and W. Greiner. Solution of the Dirac equation with two Coulomb centres. Phys. Lett. B, 47(1):5 - 7, 1973.

[86] A. Nissenand and G. Kreiss. An optimized perfectly matched layer for the Schrödinger equations. Commun. Comput. Phys., 9(1):147-179, 2011.

[87] F. Pascal and J.-M. Ghidaglia. Footbridge between finite volumes and finite elements with applications to CFD. Internat. J. Numer. Methods Fluids, 37(8):951-986, 2001.

[88] O. Pinaud. Absorbing layers for the Dirac equation. J. Comput. Phys., 289:169-180, 2015.

[89] P.-A. Raviart and J.-M. Thomas. Introduction à l'analyse numérique des équations aux dérivées partielles. Collection Mathématiques Appliquées pour la Maîtrise. [Collection of Applied Mathematics for the Master's Degree]. Masson, Paris, 1983.

[90] J. Reinhardt, B. Müller, and W. Greiner. Theory of positron production in heavy-ion collisions. Phys. Rev. A, 24:103-128, Jul 1981.

[91] E. B. Rozenbaum, D. A. Glazov, V. M. Shabaev, K. E. Sosnova, and D. A. Telnov. Dual-kinetic-balance approach to the Dirac equation for axially symmetric systems: Application to static and time-dependent fields. Phys. Rev. A, 89:012514, Jan 2014.

[92] Y. Salamin, S. X. Hu, K. Z. Hatsagortsyan, and C. H. Keitel. Relativistic high-power laser-matter interactions. Physics Reports, 427(2-3):41 - 155, 2006. 
[93] S. Salomonson and P. Öster. Relativistic all-order pair functions from a discretized single-particle Dirac Hamiltonian. Phys. Rev. A, 40(10):5548-5558, Nov 1989.

[94] S. Selstø, E. Lindroth, and J. Bengtsson. Solution of the Dirac equation for hydrogenlike systems exposed to intense electromagnetic pulses. Phys. Rev. A, 79(4):043418, Apr 2009.

[95] J. C. Strikwerda. Finite Difference Schemes and Partial Differential Equations. Society for Industrial and Applied Mathematics (SIAM), Philadelphia, PA, second edition, 2004.

[96] S. Succi and R. Benzi. Lattice Boltzmann equation for quantum mechanics. Physica D: Nonlinear Phenomena, 69(34):327 - 332, 1993.

[97] J. D. Talman. Minimax principle for the Dirac equation. Phys. Rev. Lett., 57:10911094, Sep 1986.

[98] M.E. Taylor. Pseudodifferential Operators. Princeton University Press, Princeton, NJ, 1981.

[99] B. Thaller. The Dirac equation. Texts and Monographs in Physics. Springer-Verlag, Berlin, 1992.

[100] E. F. Toro. Riemann solvers and numerical methods for fluid dynamics. SpringerVerlag, Berlin, second edition, 1999. A practical introduction.

[101] S.V. Tsynkov. Numerical solution of problems on unbounded domains. a review. Applied Numerical Mathematics, 27(4):465-532, 1998.

[102] E. Turkel and A. Yefet. Absorbing PML boundary layers for wave-like equations. Applied Numerical Mathematics, 27(4):533-557, 1998.

[103] Y. V. Vanne and A. Saenz. Solution of the time-dependent Dirac equation for multiphoton ionization of highly charged hydrogenlike ions. Phys. Rev. A, 85:033411, Mar 2012.

[104] L. Visscher, P. J. C. Aerts, O. Visser, and W. C. Nieuwpoort. Kinetic balance in contracted basis sets for relativistic calculations. International J. Quantum Chemistry, 40(S25):131-139, 1991.

[105] H. Wallmeier and W. Kutzelnigg. Basis-set expansion of the Dirac equation without variational collapse: Numerical test of the forth-back free-particle Foldy-Wouthuysen transformation. Phys. Rev. A, 28:3092-3094, Nov 1983.

[106] J. C. Wells, B. Segev, and J. Eichler. Asymptotic channels and gauge transformations of the time-dependent Dirac equation for extremely relativistic heavy-ion collisions. Phys. Rev. A, 59(1):346-357, Jan 1999. 
[107] L. Yang, D. Heinemann, and D. Kolb. Fully numerical relativistic calculations for diatomic molecules using the finite-element method. Phys. Rev. A, 48:2700-2707, Oct 1993.

[108] Y.Q. Zeng, J.Q. He, and Q.H. Liu. The application of the perfectly matched layer in numerical modeling of wave propagation in poroelastic media. Geophysics, 66(4):12581266, 2001.

[109] C. Zheng. A perfectly matched layer approach to the nonlinear Schrödinger wave equation. J. Comput. Phys., 227:537-556, 2007.

[110] T. Zuo and A. D. Bandrauk. Phase control of molecular ionization: $\mathrm{H}_{2}^{+}$and $\mathrm{H}_{3}^{++}$in intense two-color laser fields. Phys. Rev. A, 54(4):3254-3260, Oct 1996. 